



\title{
Aller Praktik Grossmutter
}

von

\author{
Johann Fischart.
}

Abdruck der ersten Bearbeitung (1572).

\section{Ha $11 \mathrm{e}^{\mathrm{a} / \mathrm{S}}$.}

Max $\mathrm{Niemeyer.}$

$$
1876 .
$$




\section{$K 11 / 501$}

\section{$5311810 \times 1$}

Neudrucke deutseher Litteraturwerke des XVI. und XVII. Jahrhunderts No. 2. 
Fischart's 'Aller Praktik Grossmutter' ist in zwei sehr abweichenden Fassungen vorhanden. Die erste, kiirzere Bearbeitung liegt vor in den Drucken von 1572 (A) und 1573 (B). Zu dem Drucke von 1574 unterwarf Fischart das Werk einer Umarbeitung, durch welche dasselbe um meir als das dreifache erweitert wurde. In dieser erweiterten Gestalt erscheint es auch in allen späteren Ausgaben aus den Jahren 1593. 1598. 1607. 1623 (vgl. Kurz, Deutsche Bibliothek X. S. VII ff.). In neuerer Zeit ist die zweite Bearbeitung gedruckt von Scheible in seinem Sammelwerke 'Das Kloster' Bd.VIII. S. 545-663, aber ungenau und nach der spätesten Ausgabe von 1623.

Da es bei dem so sehr verschiedenen Umfange beider Bearbeitungen nötig ist, jede besonders zu publicieren, so musste es rätlich erscheinen die zweite für ein späteres Heft der Sammlung aufzusparen und hier vorerst einen Neudruck der unzugänglicheren ersten Recension zu liefern. Die äusserst seltnen und wertvollen Drucke von 1572 und 1573 konnte ich in den Exemplaren der Züricher Stadtbibliothek durch die rühmlichst bekannte Freundlichkeit des Herrn Oberbibliothekar Dr. Horner hier in Leipzig benutzen.

A. Die erste Ausgabe vom Jahre 1572 liegt unserem Abdrucke zu Grunde. Der Titel derselben (ganz schwarz gedruckt) ist unten Seite 1 zeilengetreu wiedergegeben *). Die Ausgabe ist in 40 und enthält (incl. Titelblatt, dessen Rückseite leer ist) 14 ungezählte Blätter mit den Signa-

*) Eine Beschreibung der Ausgaben A und B findet sich schon bei -Kurz, Dtsch. Bibliothek X. S. VII-IX. Es sei ausdrücklich bemerkt, dass bei drei Abweichungen in dem Titelabdruck von A die Ungenauigkeit auf Seiten von Kurz ist. 
turen 2 - Dij. Das Schlussgedicht (Dija unten und $D i j^{b}$ ) ist zweispaltig gedruckt. - Der Holzschnitt auf dem Titel stellt links einen Mann mit einer Sense dar, der auf einem liegenden Ziegenbocke rittlings sitzt; rechts befindet sich auf einem niedrigen zweirädrigen Wagen eine weibliche Gestalt, die in der Linken einen Speer hält, zur Rechten hat sie einen Krug, auf dem oben eine Gans sitzt und ein Affe steht, der einen Bogen abschiesst.

Von dieser Ausgabe ist unser Abdruck eine genaue Reproduction. Nur folgende grobe Druckfehler sind (mit B) verbessert worden: $4_{3}{ }^{*}$ ) fruchtbat; $4_{24}$ mür D] wünd; $9_{10}$ augen]

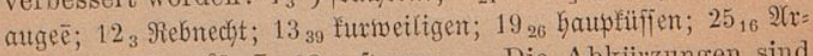
chimiften; $27_{31} z^{u}$ ] $\boldsymbol{\gamma}^{\bar{n}} ; 32_{13}$ [parcr. - Die Abkürzungen sind aufgelöst worden: häufiger ist nur è für en (47mal), v̄ für. yno ( $15 \mathrm{mal})$ und $\overline{\mathrm{n}}$ für $\mathfrak{m}$ (10 mal); andere nur vereinzelt: $\bar{m}$ für ım (5mal), e für em, $\bar{a}$ für an, $\bar{v}$ für on je einmal und 'für er $2 \mathrm{mal}$ (Scfjatgrä̈no' $15_{22}$, vno' $24_{6}$ ).

Die Nachprïung des Reindrucks hat in demselben noch drei Druckfehler ergeben, nach deren Beseitigung ich glaube fiir völlige Correctheit bürgen zu können, nämlich $\delta_{3}$ lies Rammetfeger statt $\mathscr{R}$ (mmmerfeger; $9_{17}$ ist ein nicht zu streichen; $13_{9}$ lies Excipe statt Exipe.

B.' Die Ausgabe von 1573 ist ebenfalls in $4^{\circ}$ und enthält 14 Blätter (Sign. $\mathfrak{A}-D i j)$, ohne dass jedoch die Uebereinstimmung mit A eine seitengetreue ist.

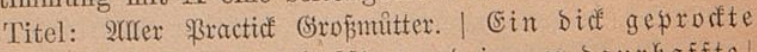
Neume bnt trewe, faurbaffe onno immer baurbafte

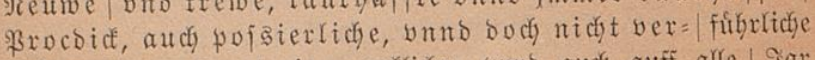
Bridfunafticats: jampt einer gedflidfen wnmo audf auff alle / Jar geredjten Raptaffeln. Seftelt burdh gît trundén, Des Stimmbjen

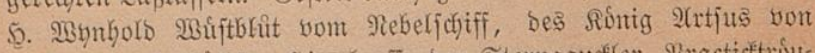
Sanbagrelwel | Góchiten Simelgaffenten Stengaudfer, Fractictivis=

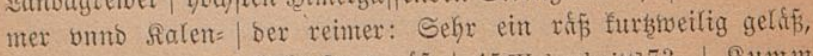

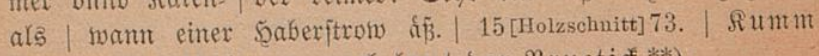
fraten Brieffelegen, nach laut ber \$ructid.**)

*) Citate nach Seiten- und Zeilenzahlen unseres Abdrucks.

**) Die gesperrten Worte sind im Original rot gedruckt. 
Der Titelholzschnitt zeigt ein gemähtes Getreidefeld; darauf rechts eine Schnitterin mit einem Rechen, links ein nackter Knabe mit einem Stabe in der Rechten. Darüber schwebt in der Luft eine menschliche Gestalt mit Fledermausflïgeln.

B ist ein im wesentlichen unveränderter Abdruck von A. Es ist nichts, was dafür spräche, dass Fischart selbst bei diesem Abdruck beteiligt gewesen wäre. Die Abweichungen sind meist orthographischer Natur, die übrigen sind sehr geringfügig. Im Folgenden sollen die Abweichungen des Textes B von A vollständig aufgeführt werden, jedoch bleiben unberücksichtigt alle rein orthographischen Differenzen, durch welche die Form des Wortes nicht weiter verändert wird. Zur Orientirung über dieselben wird es genügen, wenn man das von W. Wackernagel in seinem Deutschen Lesebuche (III, 1, 459-472) aus B abgedruckte Stiick mit unserem Texte von A vergleicht.

Seite 3, Zeile 6: 21RS A, D2 B; 7 obex mit einem Rin= den B; 10 zुmen; 21 wïrb] wirt, so meist in B; 27 Sambjtag. -

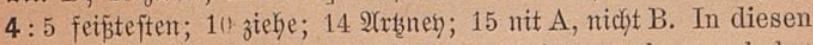
Formen differiren A und B öfter, zuweilen auch umgekehrt nicft $\mathrm{A}$, nit $\mathrm{B} ; 33$ egerbrifh. - $\mathbf{5}: 1$ vorgefn; 13 fdymedten; 25 Reütter] freuter; 31 auff ber Erben; 32 meh A, mehr B, so gewöhnlich!-6: 29 ofenloch] ofenfieçyt; 38 wa A, wo B, so immer! $-\mathbf{7 : 4}$ bex gaffen; 33 unberwoxffen. - 8: 13 Ritchenzeblex. -

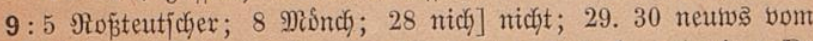
Leuffer; 33 Pater nofter. - $10: 5$ abuociern $\mathrm{A}$, abuciern B;

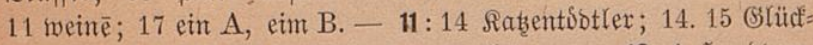
fteuber; 23 gebencfet; 27 humb wnd fateren. - 12:1 5anbiver= fchender; 7 Sumnenfeger; 8 fegten; 29 Riebhumierer; 31 Winblen weicher. - 13:8 Streubleinmadferin; 12 etliche; 17 leger opffs; 19 Demm (bem)] Dem. - 14:4 Diebijchen A, Daujctijchen B; 20 Spectfóftilern; 21 Bogelbefummeren; 29. 30 befinmmert; $34 \mathrm{eh]} \mathrm{ehe.} \mathrm{-} \mathrm{15:14} \mathrm{fumetziger]} \mathrm{Sumerziger.} \mathrm{-} \mathrm{16:16} \mathrm{ibn]}$

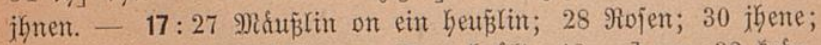
35 frautbonnen; 36 fajirt. $-\mathbf{1 8 : 1} 1$ brult; 13 an] am; 32 froku= tern] feuttern; 37 meift. - 19:2 ftreicft; 4 abfiel; 13 jm] ifm; 22 Baurentrieg; 28 eilff; 30 bie Geyligen A, ber Seiligen B. 20:15 [djarpff; 26 Sohn] Sonm. - 21:4. 5 der jein jajlegel 
fraf; 8 Simon; 11 wadflende; 28 wirftu. $-22: 9$ jmen] imren; 13 reit; 14 \$Bintermon; 17 janct A, S. B; 22 jr] ibre; 24 \$Bein; 25 Ituff S. Martins; 29 eilffe; er A, ers B; 36 ledf ein A, ledin B. - 24: 15 jafwarbeen; 35 Engelijche. $-25: 31$ fleine A, fein B; 32.33 bil Manner. - 26:6 Misndy; 21 Ifumberm; 32 getteriert. - 27:4 3ngern; 30. 31 5ुoftereidy; 37 Raiger. 28:1 5igaw; 34 2trintey. - 29:1 Iheophraftich weifen. Efifen (Bno fehlt); 2 Deß̈gleichen; 12.13 Fobagrammifchen; 19 żiehe; 29 zul leb̧t; 31 aufferifehn. - 30:3 fitegn; 11 liegen; 26 jternen

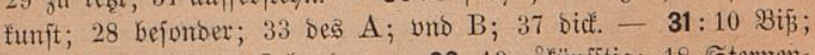
34 netws; 35 hulben] humber. - 32: 13 zúfünffitig; 18 Sternen= Docter; 19 \$seinbolde Seinfultt; 21 Eultus.

\section{Wilhelm Braune.}


Xrfer Bractiof (S) róntüter.

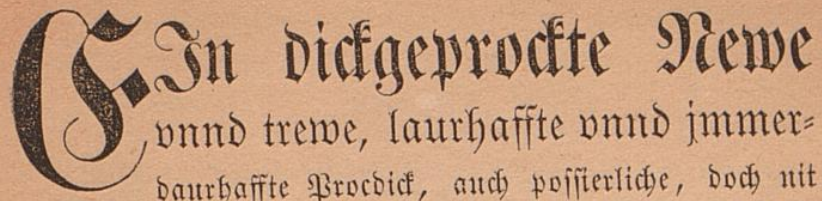

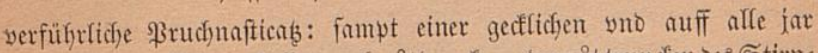

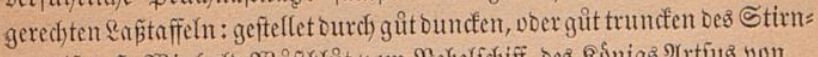

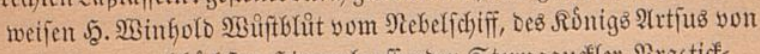

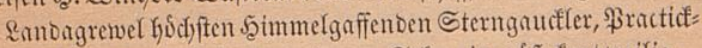

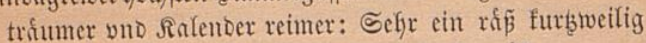

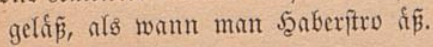

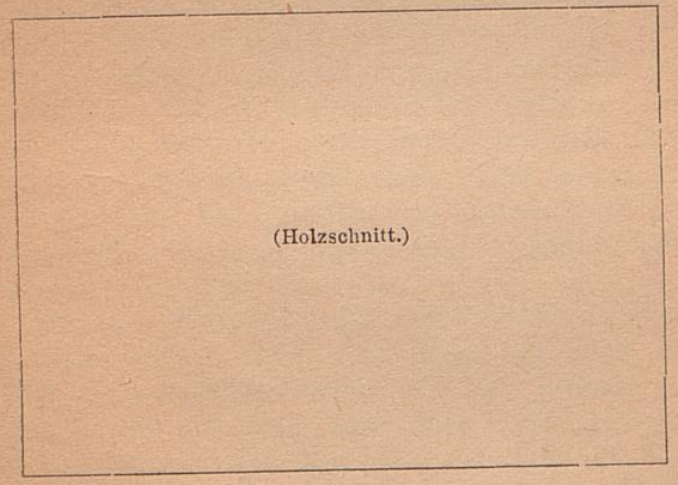

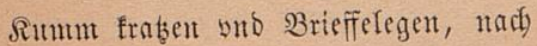
raut ber \$ructicf.

M. D. LXXII.

Fifdart, Frattit. 



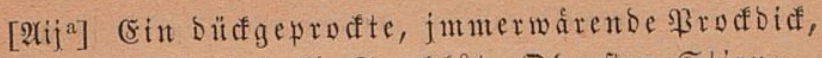
Dutd Samingold Semblut, Dberiten Stiru= maujer Ronigs Sorgantius geprodt, or= benlidy wie mant bie Satw bab, who ben Mift lab.

21

Qz man bas Gar zeidnntet mit einem gelegtent

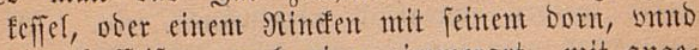
vier butfeifen, auth einter zimmeraxt, mit ange=

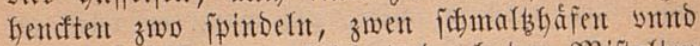
zween trifg, warb bifes geprodstiziert, Durdi bent Miftaltent

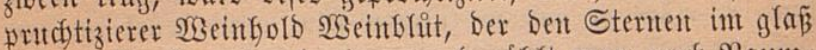
fach, auff alle volgento jar, vnto záblt man nach) Raum=

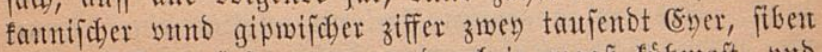
Gunoert Bratwürit, zwey ynto achzig maañ fitbmof́t, vno Galt fid wie wolget.

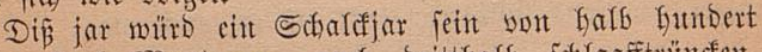
giten fant Montagen, anth) brittbalb idslanfftründent.

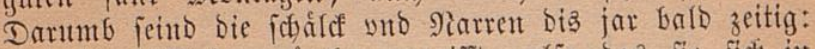
Bnd bie ruffitt bent gentusen vergifft, aljo bas fie fich itt bie ftátt vnto bátuer zulleben werbent begebent. Der Siomer

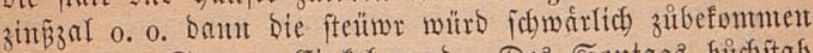
jein. Der Sounent Sirfel runo. Dez Sontags buchítab fitc) man auff ben Büufften yno ftuben, mit $Q$. S. iift

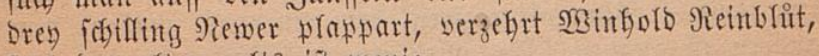
iagt bes altent geltz iit wentig.

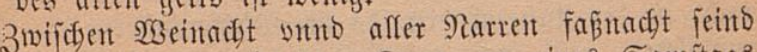
wngleiche wodbent vant tag, Damt ant eittes Samitags abent fam jentem Sdynetber ein par ftritmpin ziuplebent, ba er zà vor eint gants wod war mitsitg gefeffent. 2(ud) witro ettlidsent lieben Menjoen bie nadyt zü furb, wno ber trig zü lantg jeit, Dargegen bent faulent arbeiterit, ber tag zit lang, bie nacht zut futrb. Die gutlden zabl, erzeigt fich bey bent arment idmal.

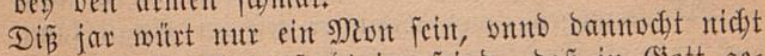

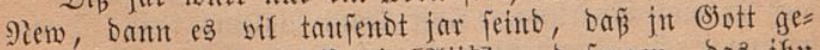

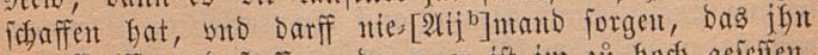
ber 2301 fwerd freffen, bant er ift jun ziu hod geicifen. 
WBant ber ફ̧immel fállt, io werbent alle Bógel gefantgent

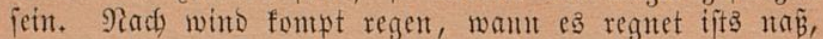
glaubit bu bas? Siebeledst jar, madyt futd tbar gar. (Eometen madyen vil Fropheten, vno jagen alf von tobten, comede mein feifteten. $\mathscr{B} e r$ lang nagel bat, mitrot ein yortbeil zufterben Gaben, buch ben grüntoigen nidgt wol befommen, aber bie Sedilabichneiber mol fromment, io idneiben fie on ein fingerfut nit in bie funger. Soroffe

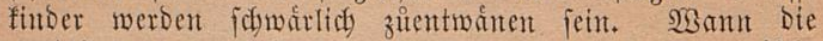
ntatintiteten war finto, fo zief) feinter feine finto. (E) wajicht

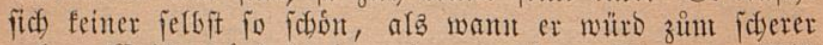
gehn. Weifie 5̧and jeint gentig gemaichen. $23 a n t$ ber Mijt

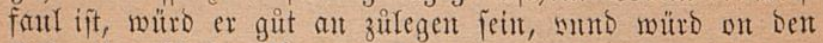

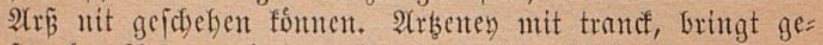
ftantf. 2(rbeney it gemein, fan nit müblich jein. Dant wir baben nidst gleich gemein fleifid blut wno bein, ein

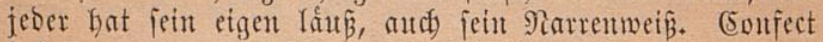

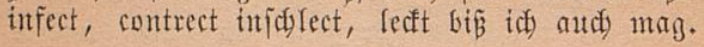

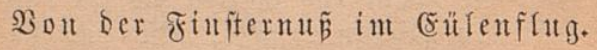

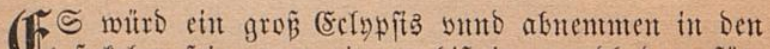

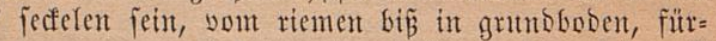
nemlich want mant bil betrieget vno Yieget, ba witro auff eim theil S. Mantgolt regien, Dem einent zit idhaben, bem

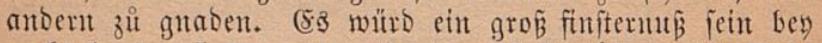
nadbt in ber finftermetten, ba bie Praffenfafferin bie liedst

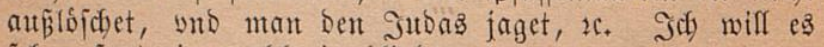
febent jagt ein mabl ein blinber.

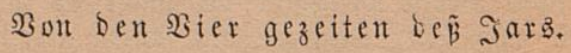

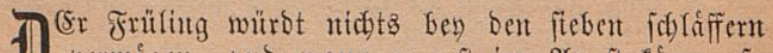
1) wermogen, who waut er erít im Atugit fáme, jo Giế er wol Spátling. Auff Sant Báftins tag, ift ber fritlittg nach. Band biemeil auff Den Rarireitag jedermant wifl flaben wnb Eyerfáz efien, witro ein eyerbritch muifen 
yorgeben, und wolfeile it Den Eyeridhalen entitehen. Die

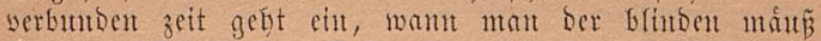

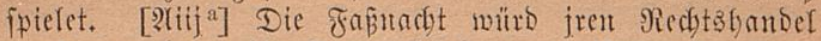

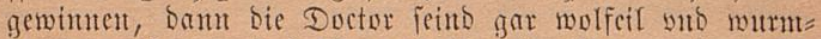
fitchig worben, whto belffen erfalten bent Farrentorden. In ber faintadt witro ein theil ber welt fich verfleiden, bas= mit fie bas antor betriegen. Mii ofy ichaben, fo mads id) sutd) mit.

Smt Sommer ift tutfent cin fommen, who wäto am

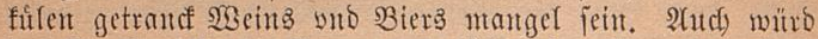
Das Dorff waffer im Brtutten fo fíz vno gefdumad werben, bas mandfem büritigen Schnitter ein falter trund waffers

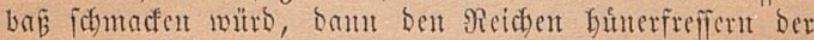

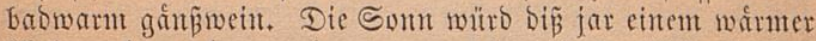
icheinent baun bent antorn, angejeben bas etlidse in bent

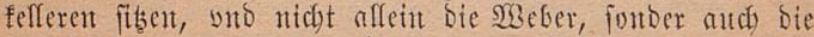
weiniputler. Daß̉ abgemtint grấ ynto bie eingeidnittent fruteft witrit nidft mebr wadjent. Sm Sommer witro

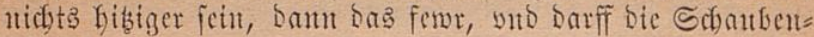
Gut berbrenten, want man bas fewr bamt antmadyt. 2utch werben bie floh ben 23 eibern faft vber bie fntie fteigen,

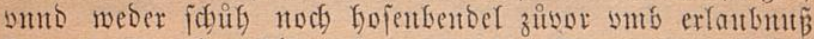
fragett. SBey bent flokent im mittel bez Sommers, ynt bent

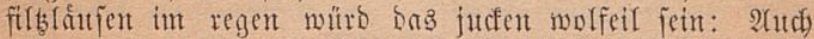
werbent bie weiber bie idwarken Nentter mit ben blofien

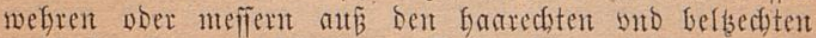

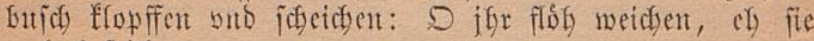
eltch) bejetchent.

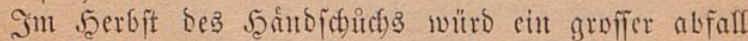

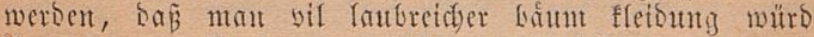
ligen jeben auffi erben, whb alio nacfent zoild antoen werbent. (53 mult bi wärb utan bie tratben yor ober nady ablejen. $29 \mathrm{cr}$ zit viel Moit einjoütt, verfutipif bie Nieftel nit. Der auff= gantg Des Meftharbi mit bent NBeinmant milto cht ab=

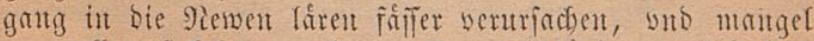
ant yolfent jedefen matben. Int weinlantoent mitrd bas

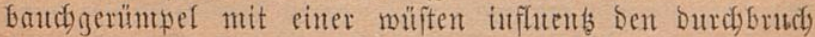
bringen, aljo bas mandfen ber weg zillantg milto jeitt, 
etwantu nut bey bent nedfiten meinjtof niber zut fitsen,

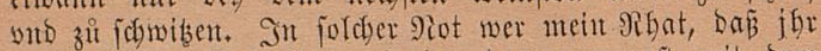
eud nticht auffinefterten, fonber bas wanmejt mit bem

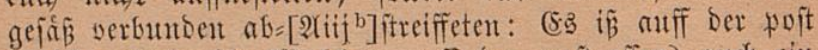

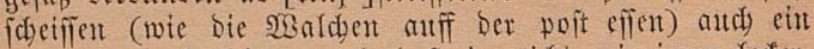

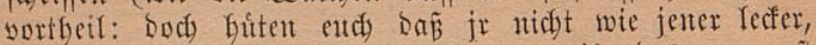
euch felber it ben Ermel idheiffet, want jhr bas mamme th nicht gentig an end balten. Dent beiten Moft würb ber gentein Mant am liebitent trincfen. (5:3 foll in biejer zeit

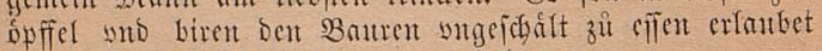

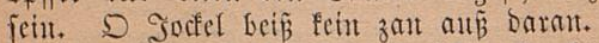

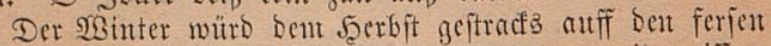

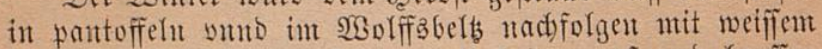

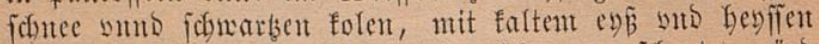

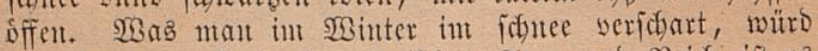

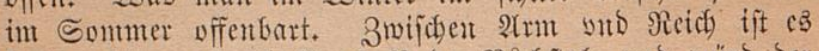

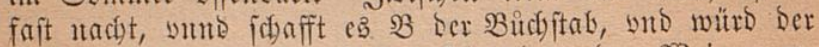

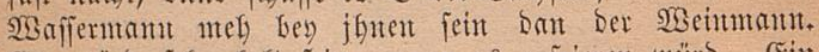

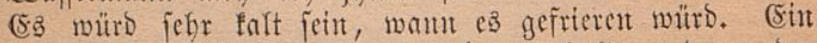
becfelfatb würb bent Rarren watuter baltelt, Dant Der soppelgebátbelten bed bent thoren fitben wno fammer vber cintanber, id) neim fie suto bielt eim ein fitut fonthall. (5:3

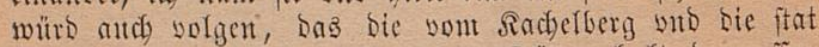
offen mit bem feutrigen 2lfpect som Dïrren bols bermafien erzultut werben, bai man fid leidhtlid an juten verbrentuen mag: Derbalben fie biffich in efrent gebaltent fein, fonber= lich sout weiber volff. Die mago vito frantwen, werben mit joldher cintbrünitiger lieb, gegen bent weiffen Moren yom ofentoch entzintot werben, Das zubbergen fie múfien jnen zit bem binbernt bincin blajen, fo lang bî̉ fie foüms aubipeyen, ond fid entgegen mit bibiger butut vertemmen faffen: Pfith) was ftinct alfo? Sireot bir ift ber belb ver=

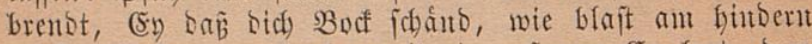
ento? Wgolan wer in ber zeit cin gefroren (E) legt, bem ift geminglidh ber magent erfalt, gleidh wie jentem bantent ber

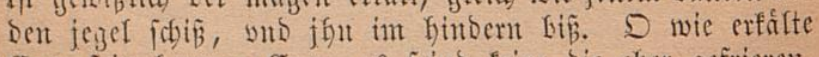
Eyer feit bawren (Eyer, ç jeint feine bie eber gefrieren,

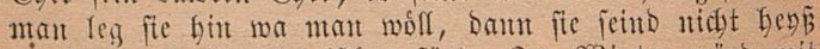

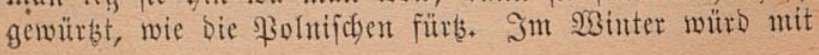


faltent Gátben ntidgt guit int bart zigreiffent jeint. Die

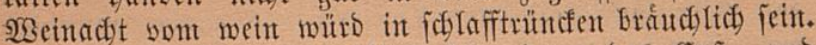

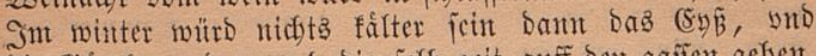
bie (Sa)th) werbent vmb bie jelb zeit anff ben gafient gebent, aud bie Rotbritiflein auffi bie najen fliegen: Daß na=

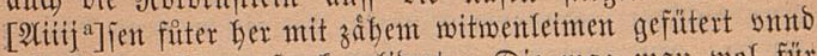
mit ftarfem baurentitrecf geltbert. Die mag man mol für Narren tauffen, bie im winter ben bels berfauffen, auff bas fie briflen fauffen, guté mir butrdis lods, feh bebarffit ein bandichuly tody.

Bon ben regirenden $\supsetneqq$ lanetent.

Je \$lanetent biź jax (wie aud alle zwar) werden sutitat jein, whto gekent nads befien willen ber fie regiert. Saturnus würo hintor fich geben wie ein itáttiger

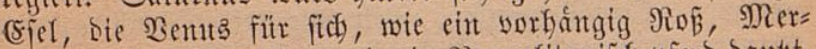

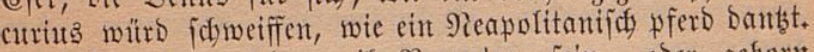
In bifent jar werden wil Regentent feit, doer geborn ober erforen. (Es jeins boje contrectationen vanto contem= plationen mit bundelen zerbrod)enent briflen in eim lárent

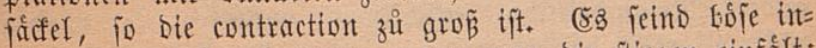
clinationen ynd neigungen want man bie fitiegen einfált:

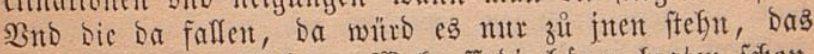
fie wibenumb auffitehn. $230 l a u f f$ bie Guter braten fidon.

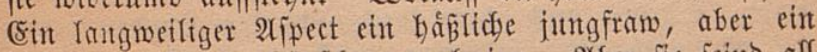

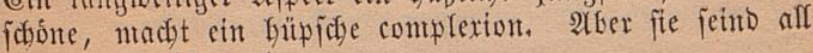
hitpid) ofh bie mein.

Boul ftand ettridaer reüth uad) Den Franeten.

1. Je Ronig werben eben bie Blaneten im bimmel Gaben, welche bie bettler, Dant auff ber Rónigent

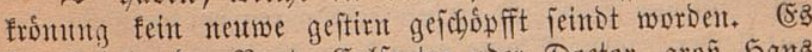

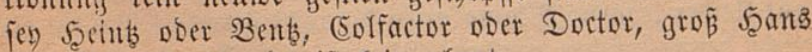
Doer flcin Saans, ba ift fein glans.

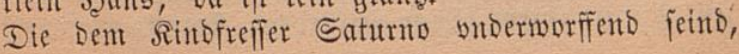


aIs alles büritiges gefintlein, wito wngerbatene fintoleint, Dấ mefr lát wetterleünige leüt, Rerdelzieber, Sambriter, Rámmerfeger,

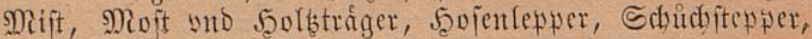

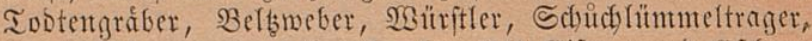

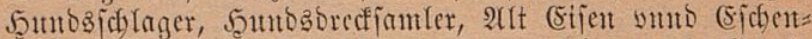

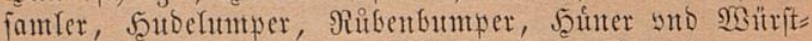
füfller, Bejemitieler, Edylegelflicfer, Steinpiffer, Biegcitter,

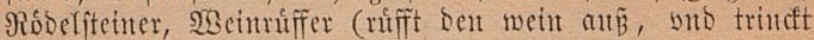
er waffer zü hanki Eeimengraber, Seitenichaber, Sount=

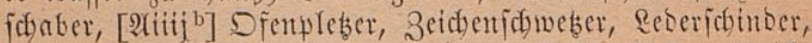
Weibentintor, WButmiamen Rramer, Salbenflicter, Reifï=

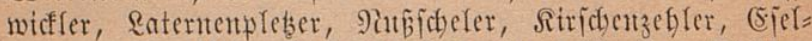
treiber, Sutapfad, Etraubleinbeff, Bartedfanter, Dritler,

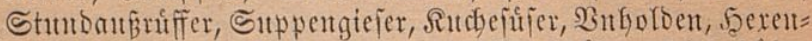
meifter, Bariflenteber, Ranbipeber, Mildyitáler, Diebshâler,

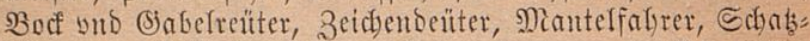
ficharrer, Eriftaflengutfer, Ecimentretter, 3willdgletter, Serner, Rimmodher, Drtbantmacher, Sitamperer, Sar=

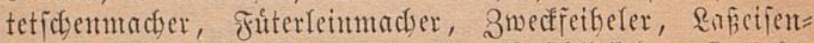
weber, Bflaiteridleiffer, Delpreffer, Dujdlitfieder, Rutteln=

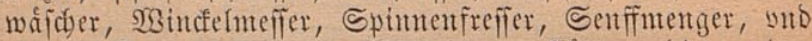
anbere Nefandfolifice brecfifflintoige vnflatter, bie weroen nicht vif recht bandel treiben, vno nidyt aflez empiangen, was fie gent erlangen, werben fich fait des Fefles ber frentberfutbung behelffen, vnto oft fiuchen laut beis̆t, 2Berbent aud jgren ipecf nidyt ben battubent geben, als ob fie es nidbt beborffen, nod) wil Thater ntad) ber Saţent werffert. Dant nidbts geminten, vif vertfon, madyt

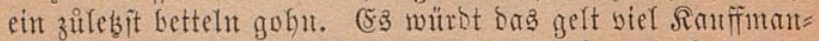
idsats bey jenten verfintorn. 2(ttd) werdent fie baffelbige

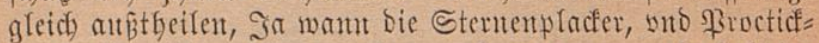
prodfer ber liggett eins werben, vnto want man cint jechtsig= járigen Baber futto, ber nie geidymibt hat, einent $23 a r j a g e r$ Der nie gelogen bat, einen Soler ber nie ift rifitg morden,

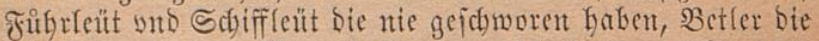
git fletber tragen, Mamaludfen bie recht glautben, Sitsent bie

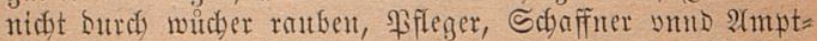
lent bie in ifrem bientit verberben, Rrancfe bie nidjt gent 
gejutot weren obn fterben, Brocutratoren die jr git fït

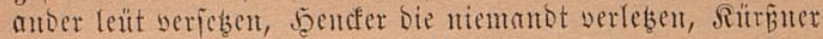
bie Dent beis nit falbent, Tagloner bie nidgt gern woltent, Daß́ bie arbeyt fichout wer vergangen, mant fie anfangent,

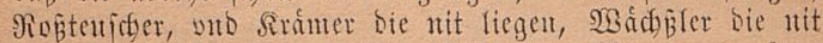

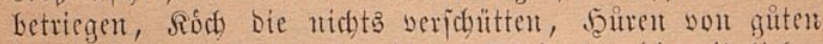
fitten, Sdyreiber bie nit rabirn, Studenten bie nit lieber

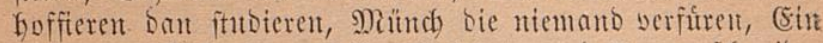

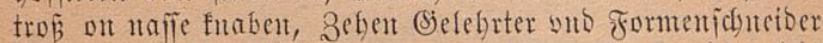

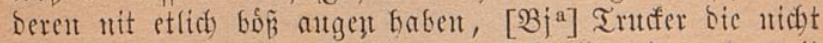
gern 2Bein trincfen: (Sin Dutbent Edheider Darunter nit ettlidbe biuden, Metger, Serber, Seitemuadber, Deler bie

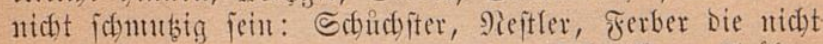
whift trigel haben, Raumengitffer, Schleiffer, Tráber, Ed)lofier, bie nit firren: WBintt bie nidbt wafier nnder ben wein ichultten: Misigo bie nicht Şáfen bredsen, Sutnge gejeflent bie nicht utidbt gern ichone Netoleit jeben, \& contra son Jungfrawent bie nidst getu idjonte gciellen idamen, 2c. Ia wan ber Teliffel geitirbt, it jul nodb. ntidht weh, $2 \mathrm{c}$.

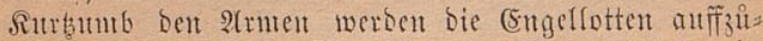

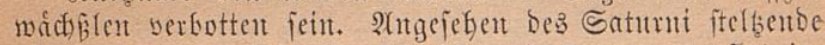
retrograbation wïto ein frippel vito frummer buflat int Epittal iterben, bno werben die Erben ntit viel vmb bas

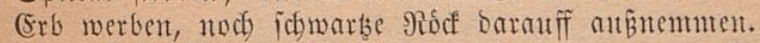

Die under Dem bloben Supiter, als bie zaghaffte ge= feffen, bie nidut viel arbentent woflen, bie eint idjefmenteint baben im rutfen, Das fie jith nich gern butfen, bie 9ientw= zeitung frager, bie lẩbricff trager, bie wat müms yom

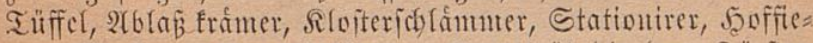
rer, (S)loffner, Secrr Eolfactor, 2(mptlen̈t bie bem fitriftent geben ein (5y, buth nemmen bem gemeinen SRant zwey,

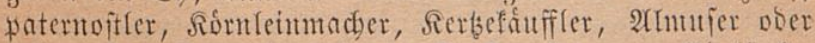

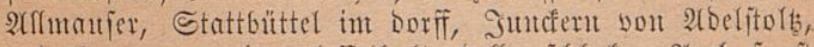

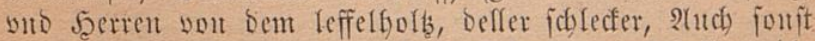

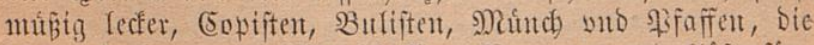

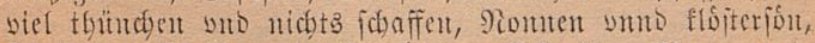

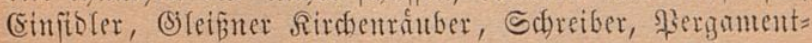
ichaber, Beutehbaber, Reticher, Rotichmeber, \$ittimen= 


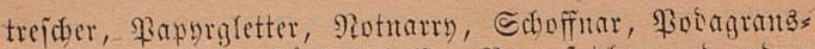

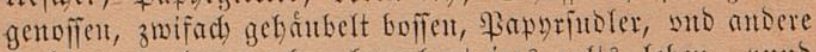
Seubler, werdent uad) gelegentent jres gelts leben, vuto ettlid mebr fiften voll brieff ober ftaub haben bant woll gelta. Die ADucaten merben Dapfier bas gelt abuocient wnd procutrient. Die Sibreiber werdent mebr gewinnent mit fanffen bund fitsen, ban ein Bott mit fantfen wno

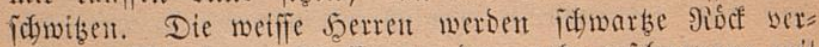

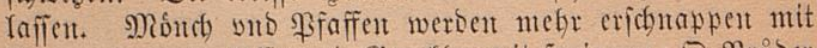
fingen, ban ein $21 \tilde{f}$ vino Bancfler mit pringen, $D$ Brîber

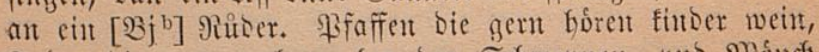

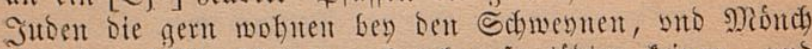
bie zil weltfich icheinen, benterer io tobstent feinten, wato

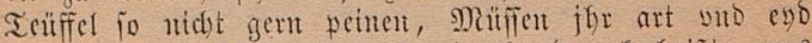
bernteinen. Saicht jebes gleid) ein bandwerd beiśt, was einten fleibet ober ipeifit, fontoer was einen nefort vno efort,

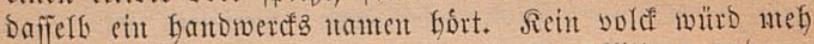
mit ber jamarben binten farben funt erfittent wno er=

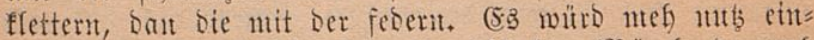

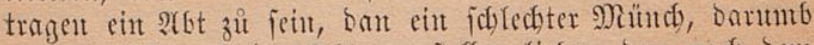
werben viel nad ben Atbteyen fteflent lieber, bat nach bem viertágigen fieber. Sd wifl lieber eins 2(ntbonier Mïncbs

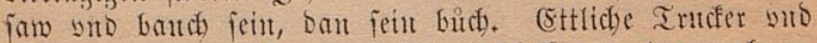
Schreiber werden fith mebrtbeils mit blofien worten ertebren. Die geifflidyent werben offt fïtgent bne ruffen, ba fie lieber ichliefien, who bettent lieber in bettent. Sie weroen jebr andaditig lejent affzeit, want es lange guitte opffer gent,

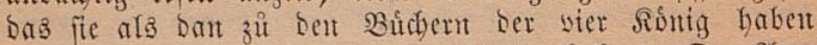

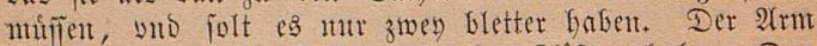
Đrif́ciants würd jein fegfentwe int ben Rlofitern baben. Den

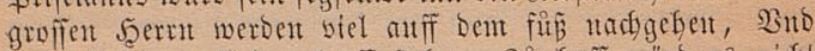
juen lieber ateff ben fopfif ftebut. Sit boff witro ez nidyt lang wol fomeden, fids idslaffen anfif bie fiftent fitedent (mant fichon golt bartmu $\mathrm{leg}$ ) bant fie jeinto eit wentg Garter bat bie febert. Die Schreiber vno Piotitarry foltent

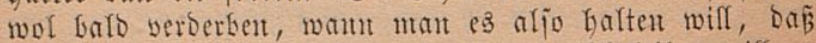

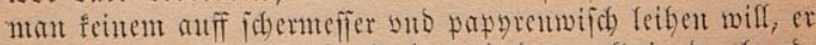
Gab ben bürgidaffit vno yfatb, who bas geft it bet hant.

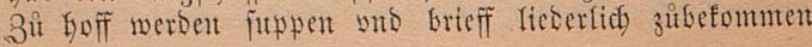


fein. Şut eud ir Goutiften, bas pobagram will zî celch nititen, jo modst eüd) wol mit belben jocfen vito frutefen

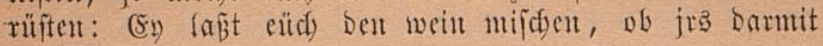
verdiliten.

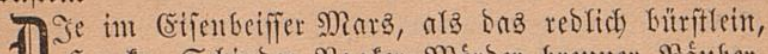

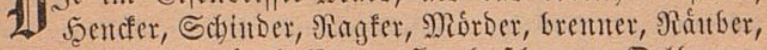
Riftenfeger, Salgenuigel, Bawrenflegel, idhergent, Delberger,

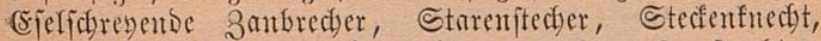
settelridter, Échupphát, Rowen, Fechter, Fanftredster,

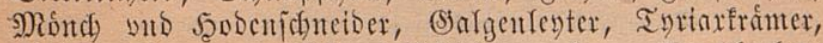

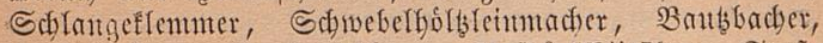

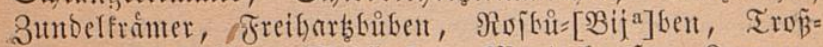
Guben, Staflbuben, Nififtucten, Marterbanjen, Rumpen=

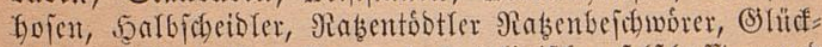
fteiber, flecfentreiber, Bangart, (Eatalinijh gejelichafitt, vnno fontit bic boje bitben feins im britten grad, wund gefino,

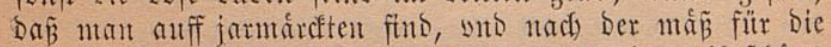
zablung an galgen binto, bie werben bié jar vil fidonts Dings Didbten vito verricbten. Alber Der Mars trimet juem

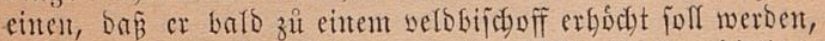
Der bent fürgefendent leüten mit ben finfen bie benebiction,

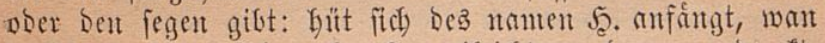

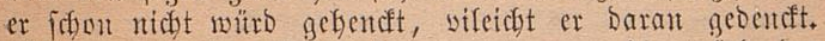
Welche nicft Der Mars fan umbringen, bie witrd ber Mors veridhlingent. Sireger, Seüter, Fechter werben fich

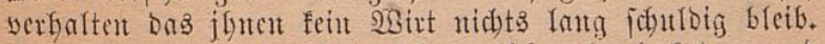
(5) witrot ein rauber frieg fein zwifdent butto Eatzen, vnto

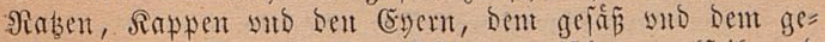

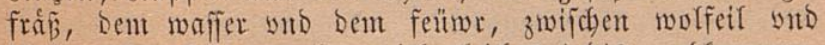
theitr. Sult frieg, merben viel gleiches tobtz 1 mbtomment, bie Dod) sutgleicl Ratiutiteten Gatten.

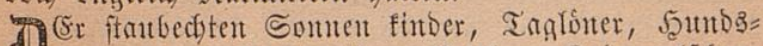

2) entwefner, Eantotzettler, Bettler, Rutberwelichen,

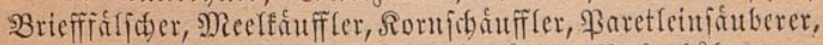
Flecfentreiber, Bierentiturer, Satntosbuben, Becfentuibent von Bajer, Sotpentuitben, Rommetfiffer, Edbitfielipieler, Sact: triger, Soltrager, Stiffelidnterer, is ba blajent want fie

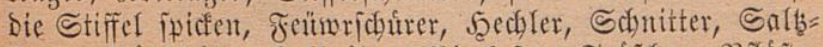

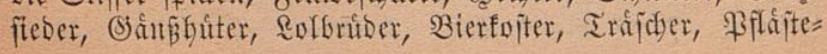




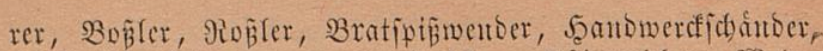

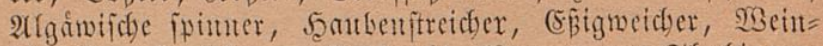

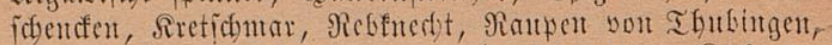

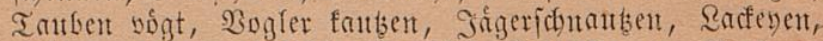
Maber, Rntienwabel idueiber, 23ectfolterfaner, Bapiren=

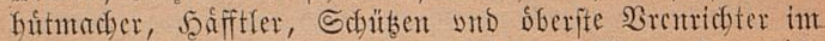

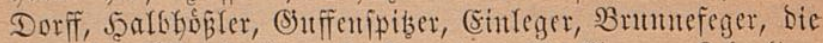

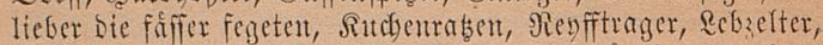
Refferidhuts, Sibutader, Strofdneiber, Rrantter, Stutben=

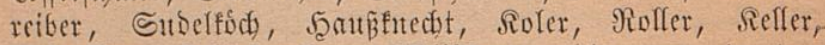

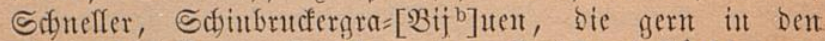
garten idslaffen, ynd afle antore jaubere beftatubte wer=

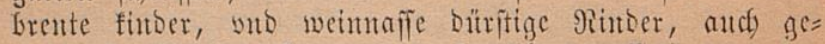
meinlich alfe bie, io gepleste Gembior auti bem rutcent tragen, ober bent bas baar zint hut auBgeft, ober bent Barfüffer orten fúbren buto latte in ber Somten erflant=

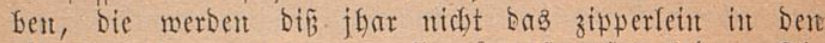
zábutut baben, want man fie zit gajt würd laben: Sie werben gert borent folagen, aber nit gern tragen: (E)

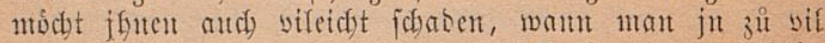
wolt antflaben, es feinb gar zarte fnaben, man fobluig jit ntit ber folbaxt eint beill. Scut enth yor bell ffeil,

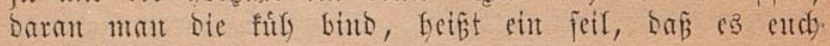
nit werb zit theil.

7. (5x 2ents mit bem bibigen ftrominten 2rich ver= 2) mante, als alferley Siaditantren, Seuren, litben,

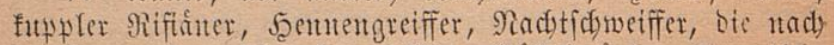

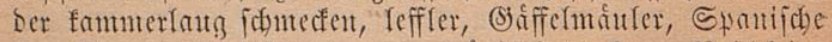
Earefïterer, Riebthntmiterer, Şanbleintruder, Brilitlein=

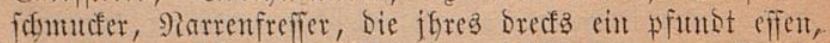
mündlein weidser, bie loblids gefellidgafft yom (Sontch,

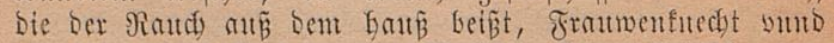

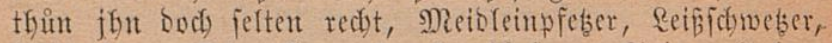
Riebtraber, Sdymollappen, Seatß̧bejem, Spinteniteder, Ramerjungen, Bodfijde Manntein, Rote Gánlein, Tüten=

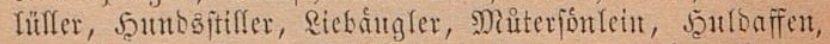
2um 2f fien atfif bem Mildbafen mit Dem pfeil Des Siarren

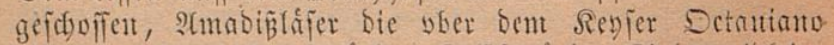

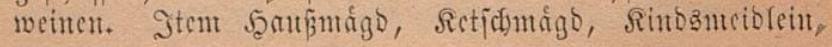


Stiffelbratute Banrenmábleit, Dantbgredeleit, Rodfentutbent

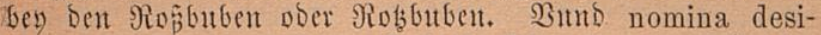
nentia in in, nament bie anff ein in fitch entent, vt Niáberin,

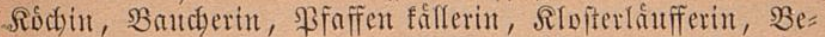

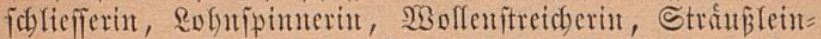
madyerin, Senffidyeberin, Babriberin, TBalcferin, 5̧ofen= itricferin, Reiberin, Eanoffemerin, SWarterin, Rlofter=

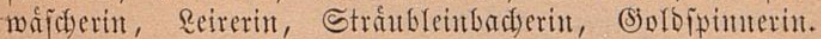
Exipe Jebamment, Scuganmen, Rantbafnedhtamutter, Rabent=

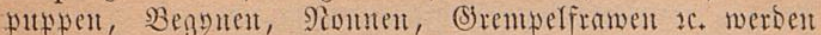

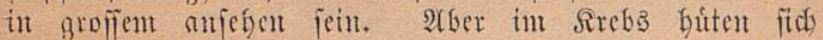
[Biija] eteliche yor bes Frantbenthojent ynto bent bucfent, want fie zit viel idyleffen. (53 werben ands feit Piontent

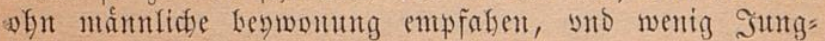
frawent Mild) geben, es werb Daun ein confteflation.

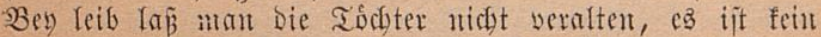

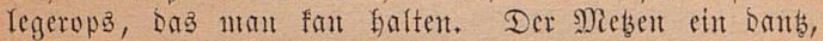
(sim juttgen fappent citt Erants, bem Spleler eit fid)ants,

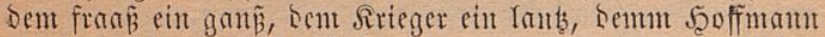
ein fudbifidwants, io ift ber reyen gants. $23 a$ bie Maigo fid) lang befinten, vnd bie frawen mutfen fpinten, ba

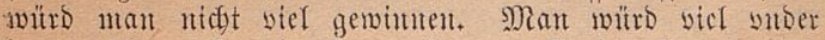
Dem tweibs voldf verdendfen, als watt fie juttgframen voer

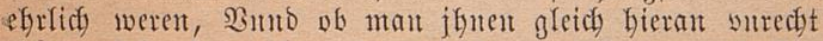

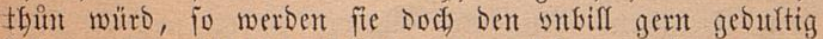

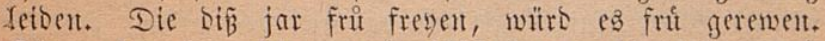
Die wol verbeurabten werben bis Jar accidentales, vnto

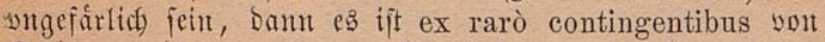
Seldentan, die es meintent werden betrogent, wie jenter lecfer,

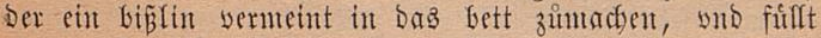
ez gar barmit, pfity, verbrenn bid nit but lappententijer am lech. Die bẩblid gementlen jundframent werbent bent fichleyer yor bem Maul haben. Der Srate von Tugent=

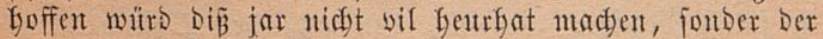
won Eugentall wno Golsburg, wers trifft, bem flapt es, Beffer ichat ban fábl, lagt ein mabl ein ichielenter ichnts.

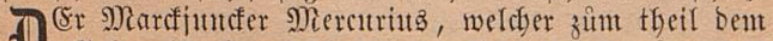
2) Seltmeldento geibbüritlein antfi Der butrit, zum theil

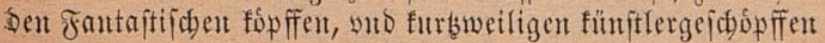


woritebet, als ben Yiegent, betriegent, Benteltreichern,

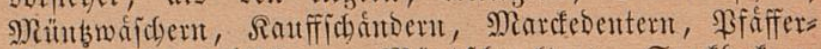

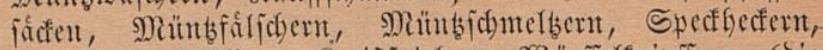

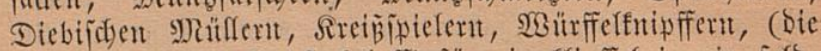
Damad) meifter Saanis fnipffit fitr ein flipffel in ein feld =

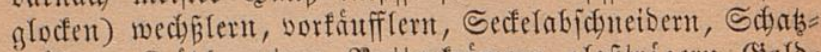

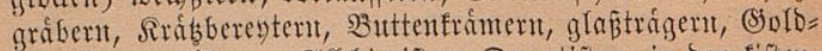

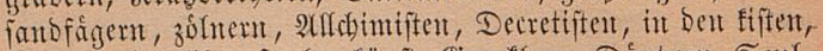
Meiften ber fiben faulen fünt, Sautefern, Dánbern, Sent= gângert, Nardiatugent, Brenteifenichteibern, Edymerb=

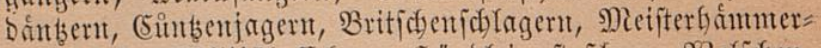

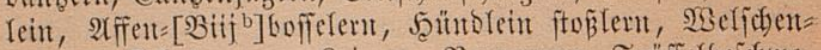
geigen, Shalmeyem, Reirent, Bergrebert, Teüffelbeidswe:

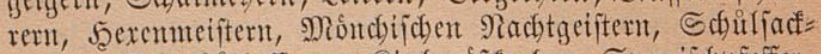

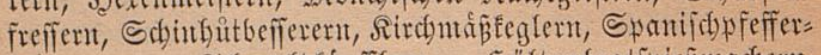

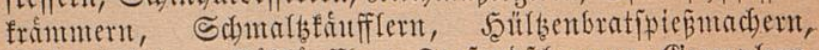

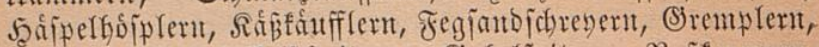

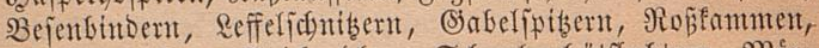
Mudenmablent, Spidnablem, Sdyaubenfütfledtern, Mcos: belfuedsten, Spedfúlblein, Sterbern, 3apfenipitbern, Teller:

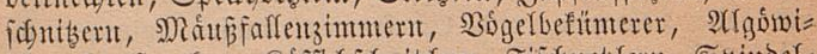

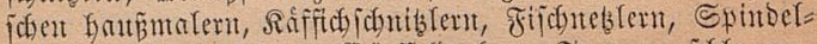

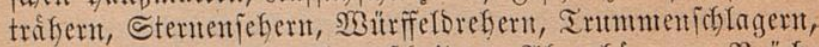

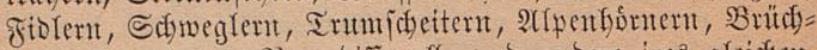

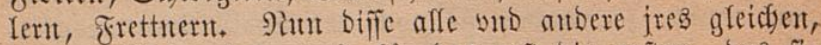
warnet Nercutrits, wnb fúr bas erjt bie eriten, das fie

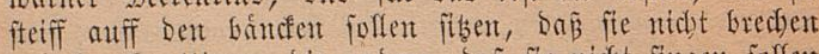

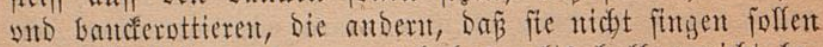
wamt fie lieber weinetent, wnd bes gelts balben nicht be= fümmert fein, fonber nutr vmb mein, brs gibt git thatbent ein. (E.3 werben bie Thicblentt an bent baument nidgt bald erfamen, ober bas pobagram friegen, bant fie bratthent

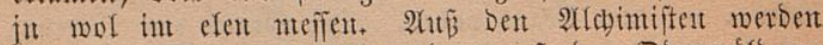

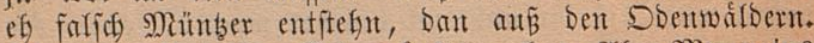
(53 witrb vil bieb auti jarmardtent geben, 20ber Mercutrits trowet ju bey jrem leben, bas fie nicht bleibent befleben,

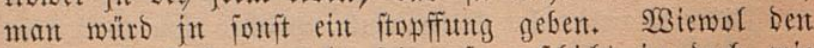
biebent alfe fleiber geredst jein, io gejohidst jut body wie ben $2(f f e n$, bie mant in ben itiffeln fätgt, wno barnach ant 
bie fetten Gaingt. Den bieben whïro man nidyt lautg trawen. Der furiter ftern trowt bem Spanijolen pfeffer, o jr Rand = frámer lautfet, jectst baz ir vorfauffet, Die bautent baben ftarfe Ejelgzungen, bie murb idsmedf ibn ponit nidbt, fite

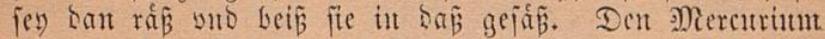

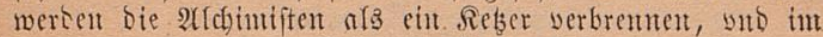
grumb ben Lapidem Spittallauficum funden.

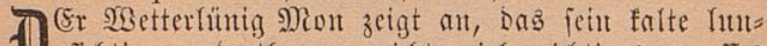

21 jidbtige unberthanen stidyt viel ridytiges groffes banbels treiben werben: als ba jeint Siarren, Stocf=

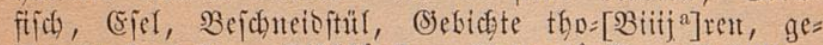

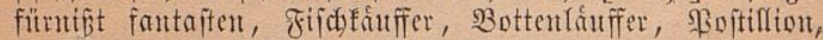

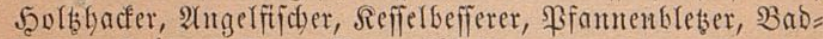
freber, Sirábenbefierer, Dergenfübrlent, fumetziger, Jacobs=

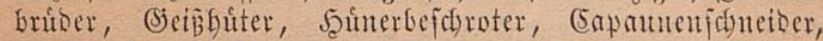
Jenterplebser, Sunbawer, Thündser, Tadbecfer, Sdjutto= leinleger, fiicherbuben von Strä̧burg, Bilger, 2Balfart= Iautfer, Bieriautfer, Rubenichreyer, Brotmáer, Scheren= (d)leyfier, Ihumpfeiffer, Edhnefengraber, Reberidaber, Rartberren, \&angent bifillierer, Efiffterer, Brntutenidópffer,

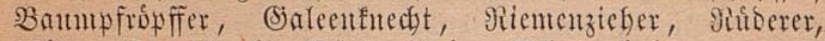

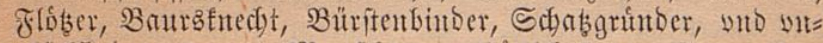

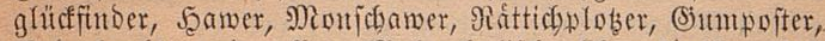

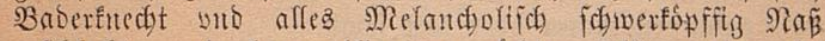
geidfledbt, who langweiliges gemádyt: Dije werden in grofiere Retchtfumb eintilden, bant fie empfinden, ynt ben fidimmel wol vom gelt treiben. Sie jollen bey leib nidat bie alten Schich binwerffen, fie baben dan newe. Die Fijofer werben zit lanto nidyt gerbaten. Die in bie Rrebslocber greiffen, werben ein Menidhent hanb Geratiz

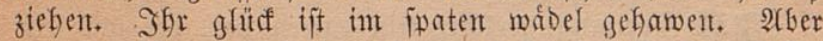
wer fan für vutghtuf, want fein bas haun voll ift: Beffer

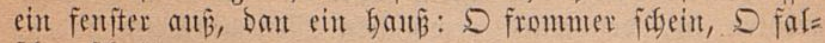
idjer iddatten. 
Bon ben XII. Mounten ynd bem eingang ber Sonthen in bie XII. 3cidsen, att jeer witrung, fautpt eitter griflifuen \&ẫtaffel.

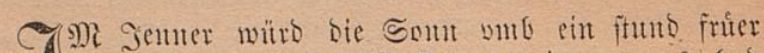

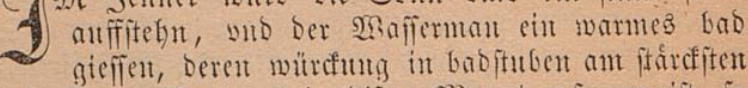

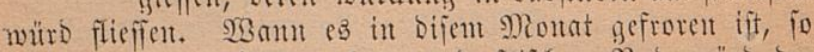

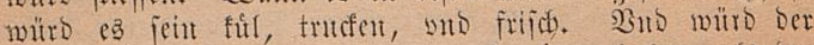
2trm gleich io wol citt futlen trund mígen babent, ala ber Reids, wiemol bie tráncf jeint vngleids. Der weî́B Edhnee müto ben snjanberen Mentident mblich feit, bat im eriten grad filt er, im anderen foutcitet er, int Drittent fauberct er. Schwarts (5ro ift golos merb, Note ift tobte, Steine ift feine, 2Beifife erb ift mun gedert. Doll iand gibt boll banto. Die altent buten fich), bas fbut nicht ber (Eyer=

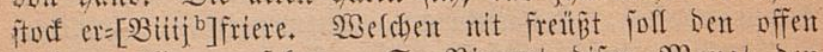
Ginderwertling anjefen. ङ. Bincent bijen Monat bent grefiten $\mathfrak{B i n t e r}$ jeino: Bno ift zil ber zeit mol ziterfeutent, welde juppent warm doer falt jeint.

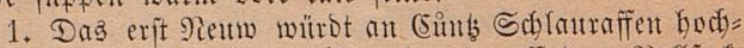

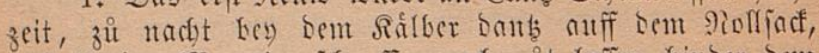
unto ift ber Mon im jobopff, anch git lafien binter bem vifen, in ber gluentoen Eachel, ba man bie fúb verbrennt, gleid) mittent im glấ, ba mant bie zungen idsleifft.

GM Sorutung, welder fein garten hat, ber foll fein

1 baum vitgebutbt lafien. $23 \mathrm{er}$ im Fifich bijes Mionat3

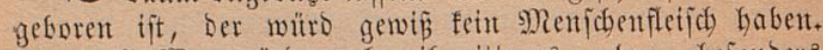
Daz falt গצee witro nod) vil zitterna geben, bejonders Den barfüffern, fie zieben ban vor Difen, ynd lejent im buid) Der Rontig yom idsellenfónig, wie Ler Ranttentbeltier

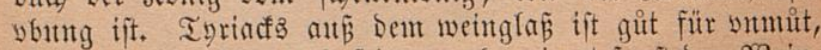

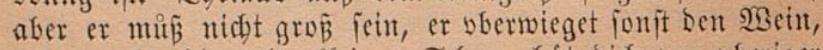
wno madyt bie wib fleit. Sdyam fut bich bor vberiger 


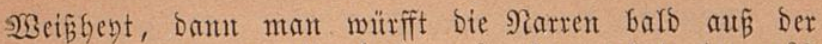

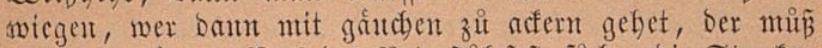
mit gecfen ágen. Bmb bes \$eteritul fejt, füchent bie Stordent jr neft, ynto fompt yon Schwalben ber reft. Mattbeis

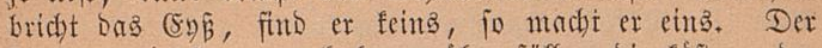
Semter vons feorntutg haben múf, füflen bie fáftent oder larent fie. (Es jagt Der Bawr eit furber formuth fer eit faur: aber fie feint felbft lautent, fite fagen mutr bon jren Mantren. In ber faften wüto bas Miserere jebr gemein jein, wie bas vluluya zwifchen Pfingitent vno Ditern.

2. Daß anber Siem würb ant falten Niontag, als bie fraw ben belb verbrant, zi mitternadbt am fonten= jichein, 3. ftund fiben minuten im (Ejclajtall, bey bem Mect= fitbel, wno ift im falb, hat junen ben fragen yno magen, whis witro gut laffen am angapffel, 2(rbentey guit auff bem ftrojact, ba ein bie feberen in hinderen beifjen.

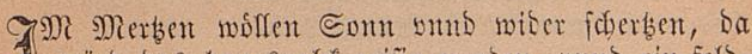

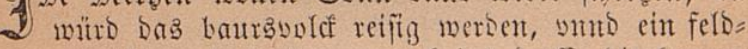
proming antichten, berbalben gemnch ins borff, bie bauten Gund iblaffen. SBant in bijem Monat mit cint ibserset Der wiber, io ftopt er jit nitioer. D bie frandfbent flief ein jeber, baun in Der gantben welt foflen viel lenith

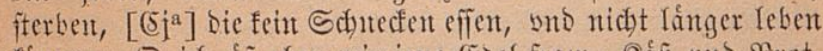

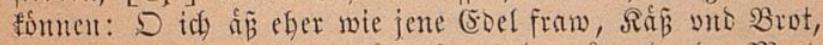
ban idb butgers ftivb. 3u anfang oder zu ent, ber Merbs fein gifitt fent. (Ein feüd)ter fauter Merbs, ijt ber bauren

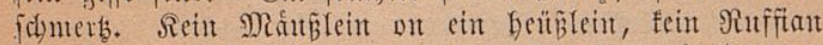
on ein putan, fein binbers on vornen, fein gios bbn Dornen.

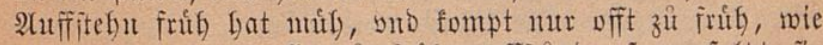
jener fraw wann fie zit frub anifitimb, fo verifalibt fie bie jupp.

3. Daß britt Reum würot an bem blawen freytag, Drey ichrit, zwo minuten binber ber thür, ba ber Jecfel

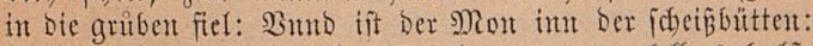

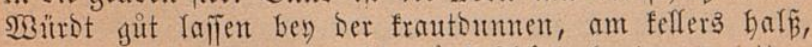
im flaben bautí, ba man bie ginn ichuth, bart am qutatter= loch, als ber lecfer bie waben beichei B, ba mant bent gelben

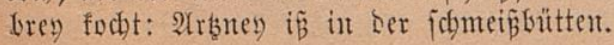

fifịart, \$raftif. 


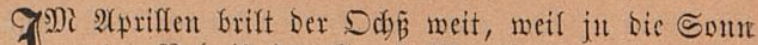
reut. Sond ift ber (Sautch Monat, bant ba lafien

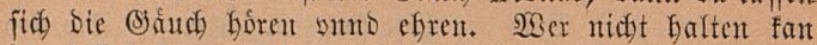
Der foll lafien. Seberman buit fich yor grofier fraucffent,

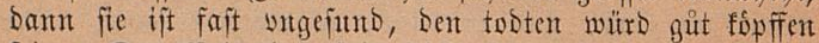
fein. Der Reitent würo leidfter vortfoument bant ber

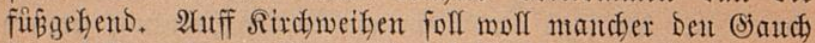
im pfeffer efien. Wiser anber lentt idwerbst, ift Darumb nit weiß̄. Eint bürrer Átprifl, ift nidgt ber bautren will, jontoer ber Priffenregen, itt jut gelegen. 21m morgent fúl im 2tprifl, madyt ichlaffen wil yno itill. Ditern iit nie on itaub yoto laub.

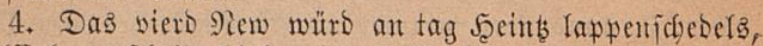
zmólff hauteniffritt bintor ber holbmutl, Da ber fribel bie hofelt fchmiert, ber Mion im fingfinger zeidhen, wmo bat junen bie oberfit früpff, würb gut lafien am lincfen of

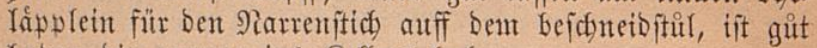
baben bie zutgen ints Rellers lods.

GMi Menen fumpt Die Sont zil zweien zwilling. 2 (baun brey hab id nie gejefen) Darumb witro niemano gent affein feint. Dent lieben mürb bie tradst faum .8. ftutto, Den záncfifdent ber tag nod fo lang werbent. WBeldye fraw bijen Monat empfángt, würo bantad bals

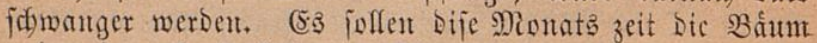
vnter bem lautb ftehen: Die gebonent Doctor merbent fait láppifich fein, ynd $\left[{ }^{\circ} j^{b}\right]$ bie alten baiut werben vil

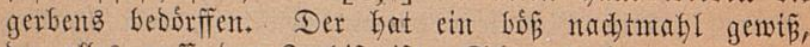

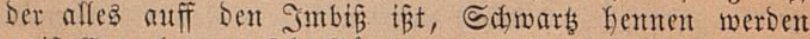
weiź (Eyer legen. Die (S) auth) werbent im felb fliegent, aber in Der Statt werdent fie gehen. 2 ser bem Sirten baß grấ abmáyet, wirb ben Edjaffen fodaben. Die Ener

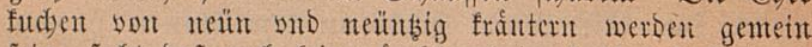

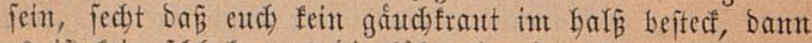

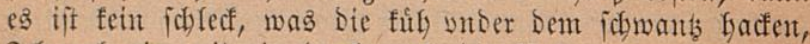

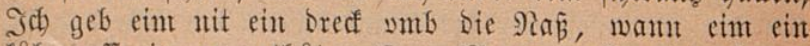
fith auff ein aug thit. Dent Meyen volf wint begert Das bautrengefinto: aber ber mefjit theil jagt ber Meyent folte fein zwijhen zwenen, nidgt zil feücht, wie múpst er 
jein vileidft? Sit ber Mey ein gârtner, fo ift ex ein gitter acferer, \& contra wie man bie wollen ftreildat.

5. Das fïnfft Nen wïrb am gelben Mittwody, als ber Ripel bie jtieg abfül mit brey idyitfiel eingejultber

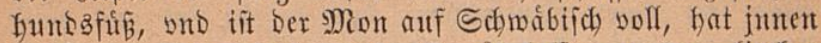

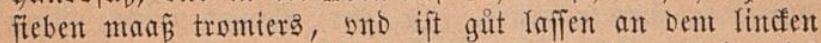

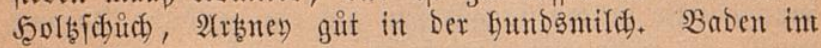
weinbact.

IM Bradfmonat mürb bas ichafficherent exit redbt

1 angebn, ban bie Son bringt ben frebs mit jeinen groffen Thoumifiden idberen, welden bie bautren zi Rieg= fels für ein idneiber anjaben, vno barnach ertrencten.

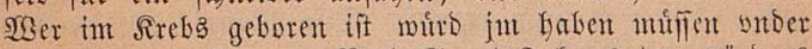
Dem ichwant liegen. Smb Sanct Johans tag witro eb viel brunft gebent. Die ichonften würo man am liebitent Gaben. Bey ben boben bergen wird man thieffe tháler finten. Barnabas ben laingitent tag laß, vnto witet jent im hew. Sanct Mebarb ift ein Máder, wno bas er nidft regen trag, er regnet iont viertzeben tag, wno nuch mehr wers glautben mag. Beg ben idslofien vnt iduntion müro ę vil ftáblents geben, So werben bie Şaffner aber eit mabl ben bauren frifg antidten. Şerkgog von Dúritberg wïrb vmbjalagen, yno bas Mildyold nad bem gejunto= brumten weifent. פ3ann es nach bem Srebs gefent foll, fo werben bie jeuten fintoer fich gehn, bie jobamel auff bie baind iteigen, bie bauptfitifen ligen zù Den fuiffen, ber bautb) zì vorberît gehen, ber 2xrich zum eriten niberifiben,

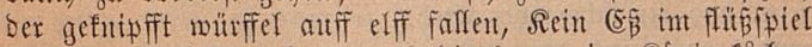
gefunben [(5ifa] werben, noch bie bonen im fisnig finchen auff bie Genligen brey Rontig tag, who bie naien frumm

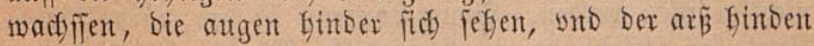
attí blebelt.

6. Das jechft siem würo auff bent futugerigen freys

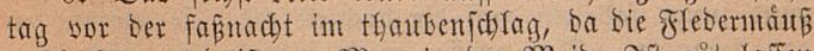

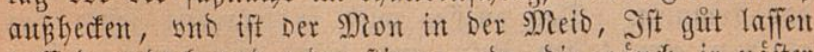
auff bem bad ynber ber fitiegen, ba bie gâud jr najter Gaben. 


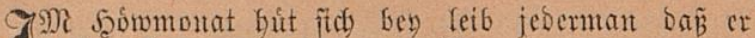

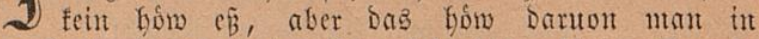
libern fingt, bas bringt. (5.3 witrot bije zeit bie Son io bod) ftebu, bas fie ntentand erlangen wutro, und io fait cilen bent lowen zuentlantfen, Das fie jhr ynd vus beiffe funtostag madent würo. Sil bem Montat werben Sient mutfent fette balben io gut fein als ein weltbun, Darumb werfft fite nidht auf bem falat ober pfeffer. 2(ud) würo Das flein gevogel umb Sanct Beits tag jo heimija werben, Das es fren mit bom grobjten bantmren bie Milds würb altí ber idulffiel effen. Berbrodsen fenjter werben zer=

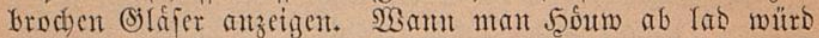
man vor groffem geftaub Die Mute nidst jefen, bie Darauff

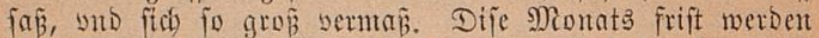
bie bornen icharfif fein, Darumb wer zornten will, gang

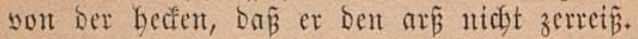

7. Dả fibent Rem mürb auff bes farnoffels tag Des fpielers, brey ftuts nach mitterntadst, vier mimuten im gold, who ijt ber Mon in eim bojen zeidjen, wan ber

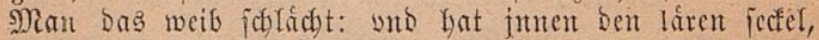

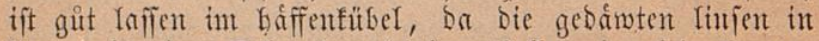
Yiegen, bie bem Edymaben jeind entfallen, ber jein farrent= máfier mit vier juttgen beglein baran verjollecfet: 2Trbुneb) git it ber fuppen, gut baben im fudjentaben, Da ber frits binein gutbt, vno man sie buto bejpribt.

gMi 2ugit gibt ea bitigig lieb ban bie Sobn geht 2 in bie juthgfram, bife zeit würo fu granjaut bey fein, bas ein fowmartier Sirebs, io man ju fiedet, gants rot

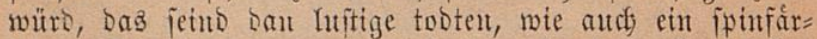
Yeint. Die in bunstagen nad) Dem bund Iauffen follen

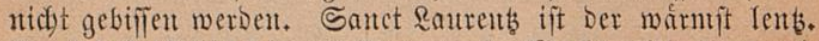

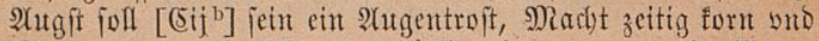
Moit. (Ë würo fid) nit beborffen bas man buth, fliegent bunto junge fábrlein aufi bie fochzeit lab, fie werben on Das fomment. Das putlffer wiro fo feürgitig jein, Das es you eim falten ftcin vito futell eifen mag erzürnt werben. Syut bich yor bleyentent piffulein, fie modyten bir zuifarff fein. Die Dürren fúlg werden meh Mild geben, 
Dann bie feişten odjen. Man witro bie fifich mit ben bantoen faben obut Reigeridmalts im bred gejalbst, (5y) lieber Iraps, greifĩ Darmit it Galf́.

8. Das adbt शiew würo aufi Eutl Sdumolnars tris, ber

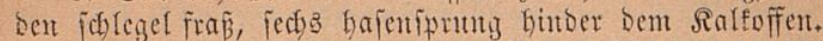
ButD ift ber tag furb mant ber Mann nidbt Daheim ift, müro gut lafien in Der flapperbücbien, bey ben aftent Weibern, zu Dem Sieman Shnetber in ber wafjer gaffen: Sit ber Mon im freba, fo gut anjeling binder fich gebent.

7. (Ex 5erbjtmonat fiteft in ber mag, ba witro er viel 2] wadelende weinenten geben. Barabs mitto auti

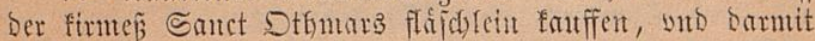
tantfent. Depffel nnto Bierent werben mit bem gegenjobeint Des itarden wintos groffent abfafl leiben. Der Santfflent glüaf würo in ber wag ftefn. Man witro nicht viel Res= hutner bmb mentig Danzapffen geben, aber viel Sdnedent id)alen würo man zablen. Sit in serbit baz metter befl, ๔o bringt es wint in $2 B i n t e r$ idneff. T2a feint Bangart ift, witrot leicht trauben abzitbrecben fein. Sit weinféfiern vno gaufelfácton, follen groffe freutben itecfert. Mant wïrb bent weint on leytern in ben Galé ablafien. Bor bem Socrbft witrot man nidyt bals moft trinden. Santet Brbans wlag vno ber Rang, witro vielen madent bang. Die yor bent burft trincfen, $23 e r b e n$ nidyt bald in eint

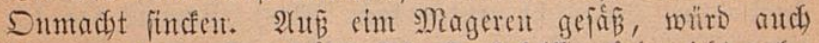

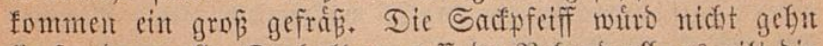
fie jey ban yoll. Derfalben auff but Nebentoroll, es gilt bir

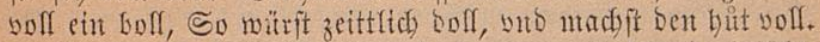

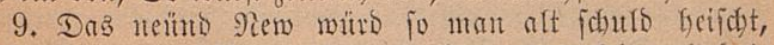
yunb ift in eim bojen zeidsen, fo ber mann fein gelt bat,

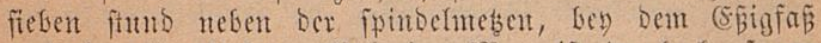
nad) Der bierglocfen. Bub ber Miont ift im bod, io ct Die geve ftổt.

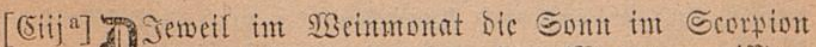
21 i it, witro es mandsem bent Magent vergifften,

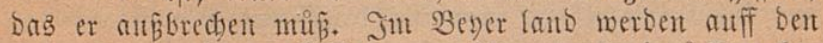
2lfern ganz mágen voll Raben madjen, im lanb zit Franden 
wirb fein flegen mefre jein, aber auß tíggen bieren werbent

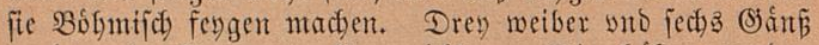
werben ein flappermandt antidyten. Die bafen werben auff bie brey fúp fiteigen, bie Gut auff bie fópfï, bie ftein merben hart jein, bie floh fdymars, wie aud fonit bie Siadtraben ynd fintere Enaben, bie bey nadyt traben.

10. Das zefento Rew würo am gritnen Mittwod im finftern loch, da bie (Ëllen ichnappen, $\mathfrak{B}$ to it ber Mon in Der geysi, hat juen bas mittel in ber glocfent, würo

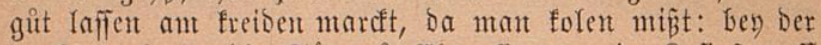

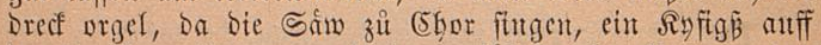

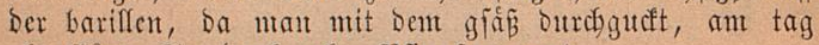
âs Sints Sambreff gehn Pringiten reut.

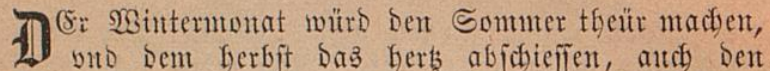
id)ütben feirabento geben. Die bimmel febern werden zì fliegen anfangen, bod nicht fo warm fein als fanct Martints gans fesern. Dem flachs würo es vbel gefn mitro er. antorit nidft gerabbredst. (E3 witro viel plitgents vno bon= nerens geben in Babituben. Die yon Sdyeinfort werdent

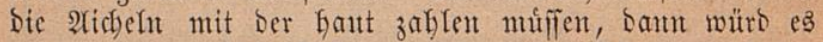
in eim giten najd pecft fein. (Es werben fid jo viel reber ipat niberlegen, bant frú auffitefn, 21tfi Ganct Martint fibt man guten win, aber ba erichrict mancher bautr uno Sintimant, Der uidbt zablen fan. Auff Nartins tag Der

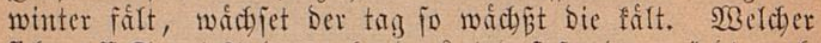
(iti) autif Sanct 2Anores abens zit tod fajt, ber witrot noch bifen Monat fterben münen, sberlebt ers aber, who jar nod) elfie barzil, jo witrst er vimb ein jar âter werden.

11. Daß eylfift Rem witro an bem tag bes würoigen Iabenbefierers, wnd ift ber Mon im idfiffelturb, ba die fram bem Seerman Den fifhberen wber Den fopff zog, zwijchent

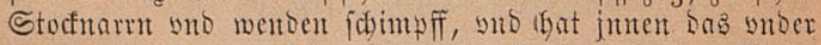
theil ber ftelben: Sit gut laffen Ginden an ber Mifit= porten, in ber vnberen 2 potecfen, ba mant bas Baljant

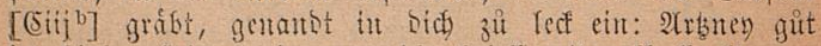
im bintoer iptegel, ba man bie briefi mit gelb figelt. 
T) $\mathfrak{M o l f f m m u t a t}$ wutro ber Eteintoof bie Soutt

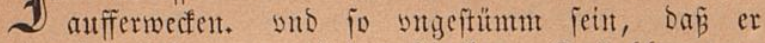

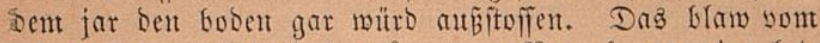
f̧immel zwey lot, Das grint vom Regentbogen vier lot, ein fuct von bem Rebel, baz allez mit eint Affenzagel

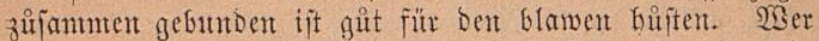
Difen Mionat utit vberlebent mag, Dem folf niemants feit

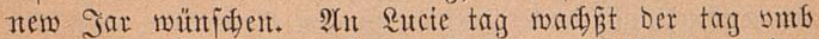

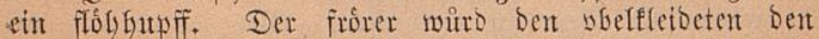

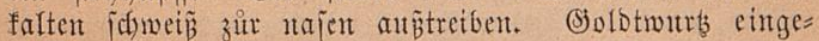
nomment io stel genilg ifit, fït bie armit guit.

12. Daß zmólfft Rew würt am tag Şeint lapp ben

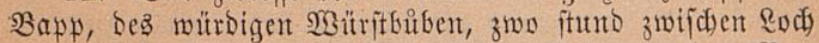
wnt Brichment. in bem (Entenflutg. Sho geht ber Ment in bie Rarpffen, in ein búpz zeidhen, baz haben bie Rarren junen, würo gît lafien am tag Seib Tobtengrabers, vor

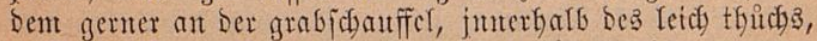

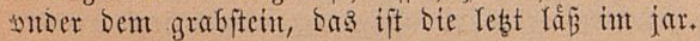

Bon früdten, Dba, SBetn, aut antoerm gentif

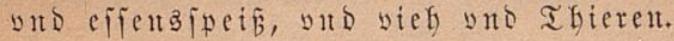

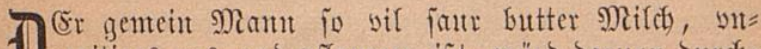

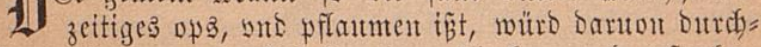

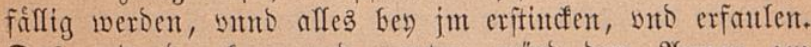
Daß getreyb, forn bno weylent witro bem 2 trment ant

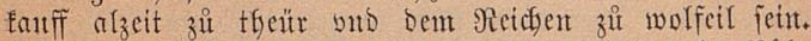

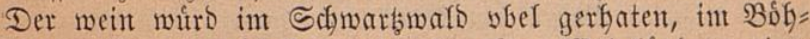
mer warb gar smbfalfen, aber in giuten $\mathfrak{S B}$ eintantern zims

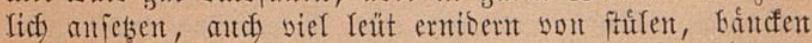
wno ítiegent. Bolle flápdent werben madbent láre táfichen,

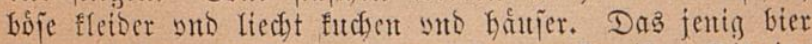
ift ant beften, barint an wentigitent wafier ift: Dis jar würd es viel terident, pflatmen, speffel wnt bieren gebent, wito werdent wol geraften auff bem obem Marcft zí

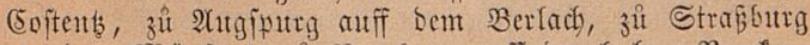
yor bem Mänjter, 3ü Bamberg auff ber Gohen Butufert. 
Bon weldyem feridsengencich viel leut bic itein im leits

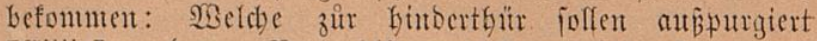
[Eiiij ${ }^{2}$ ] werbent. Butb bije zeit jagent bie altent were Dz Nautrent ant bejtent, angejefent Das Miorbel vato iteit bey) cintanter jein: 2tuch jein bie ftein gebort gut zit: márm fácflein bie bie Nontent vnber bie füßj legen, fürnem=

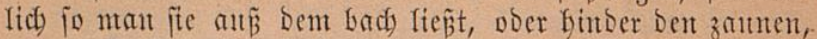
mutr auffgeflopfft ond bie fern zit ben 21potecfern geidsifft, bie wififen fie thent zit verfatffen. $23 e r$ ben weit nicht bermag, witro fich bas wafjer zu triutent nidyt beridhmebent Iaffen. $23 a$ bie Narren nticht brot effent, whitro man ben.

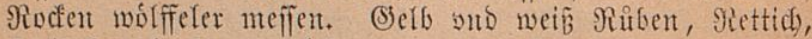

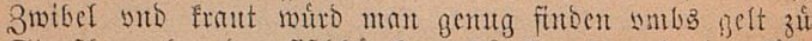

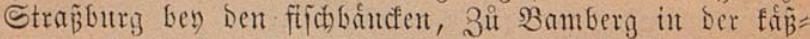
lers gaाjen vuto auti bem frantmardt. Die jomarbse fith werbent weifje Dilch geben. Dî́, jar muro das grojt

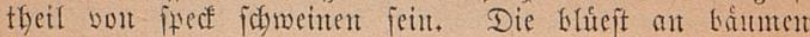

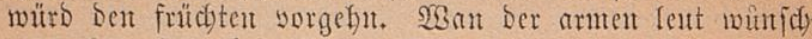
vortgeft, fo witrd ein groffer vberfltí des Rorms vuto

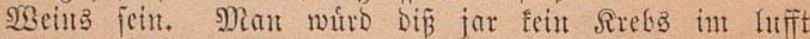
fautgen. Ein groffer mangel mürot ant biftelen jein, you wegent ber Ejel mit ben furben Dhren. 24nfi wol fiten

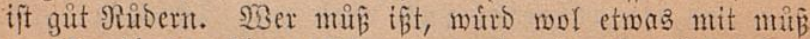
erzeblen fonten, furntemlid) want er ben brey im Mant bat. 23 anu ber ftein anff liegt witro ber bratipiz nidnt meb

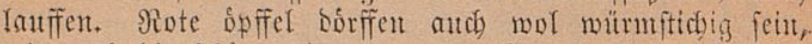

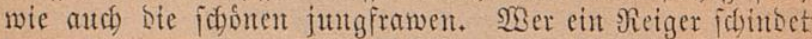
bat ein magern yogel. (S)effit in bie Erbien, io ijejt fein bonent. Drey trigig fríd), taugen auff fein Tijd), vif

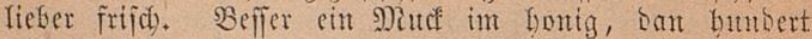
Girnautfen on foutig, ban wa nidgt ift ipeis, ba jeino

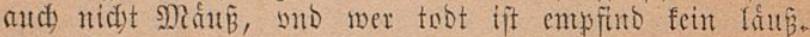

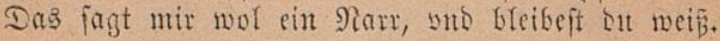

\section{Metarf, (Sold ynd Reidythut}

1] 2ts Silber mutro bent bley vorgefut, atth bem Entg=

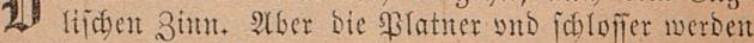
mebr eifon veridmieben ban filbers. Dns fupffer wür 
$3^{\mathrm{it}}$ groflen (Efren fommen, ban mans in viel Maints= Guitten zum Silber benthaten müro. Dą geidmiert golo mütro sngeidsmirt nicht pros baben. Daß beilig frelts witro man aufi ber gutben Múnts werb balten veto leiben, aber an ber ftiv=[(5iiij $\left.{ }^{b}\right]$ ten witro mans meiben. D but

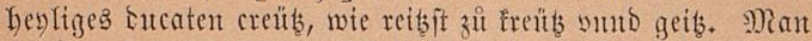
witto meh befontitten gelt3 finten, ban beichnitten funger. Die fronen werben febr getudft werben. Man mitro nidgta bald io fleiñig bebalten, als bie alten boppeln Ducatent. (S)eft mitrd bringen gutuft, aber feit futuft. Die im B̧erg= wercf graben merben meh fteit futben, bant geit grünben.

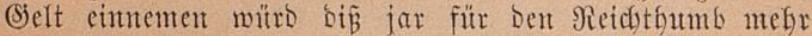

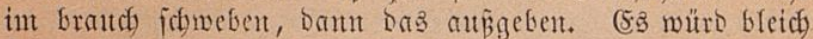
feben golo und gelo, wor jorgen, das man jhm jo jefyr nadjitelt. Die reidbent werben beffer zeblen mogen (wann fie anterif moflent) dan bie Atument. Die Alldimiftent werben ben Nercurium braten und fieden, vito in gruth ben laptom Epittal lauficum idsmieben. $29 e r$ bas gelt beracbt vmb des bojen jecfels wiflen, Den jolt man mit Mum fitflen. (Es witud git feit, Das man meb vorrbat ichaff you gelt dan son bew. Dann wiemol es theltr ift, fo effen es Dodh nidft alfe thier. Des $\mathbb{S}$. Francifict reisen

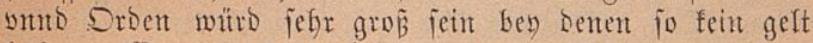
Gaben. 28 er gelt hat wurs es ohn zweiffel marm balten, wer feins bat, barfi fein jecfel barzi fauffen, nod) zu ben

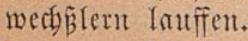

Bou Bugern erfortem glüf.

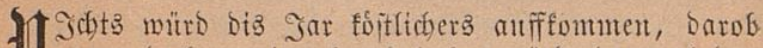
man bod) wentiger frewd haben würd, Dann ob ben vmerfofften frithten ber idymangeren jungfrawen, ob wel= d)er frutdet fleine fremo iit, io Dod nidgts foiftichers ban Der Menich auff erben erichaffen witrb. Su werben Biel= mantuter sutrd grame baar zit ebren vito einem altut ant= fehen fommen, beffent fite fid antid fremen werben, wiemol fie in ber jugento nidit fonsten bald gentig alt merben. Der Santentraib müro ben fanten Maggent nicht lieb fein, 


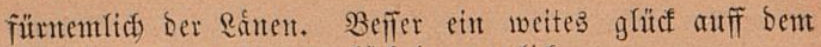

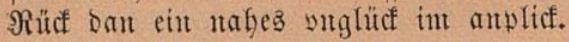

\section{(J) ewitter.}

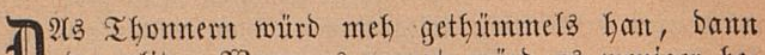

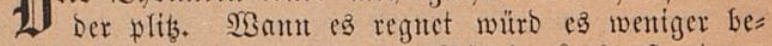
fitantbt ichưch geben. Şaltet bie Meïnd zit Gaur, Dant

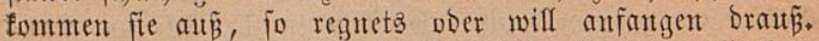

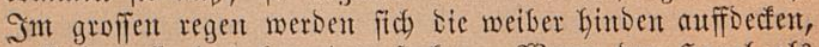

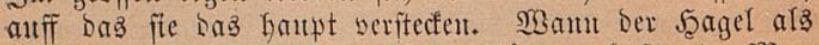

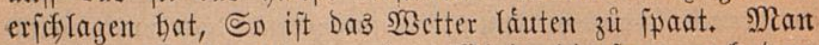
fent Das [Di ${ }^{a}$ ] wetter an Dem $2 B$ into, Die fram nach bem

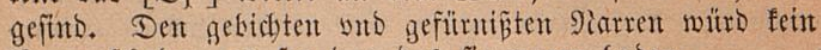
Regen fidaden, es fey bat bas fie mam baben.

\section{Bon Siationen yub Statten.}

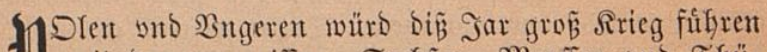

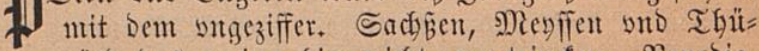
ringen wïrs bas gerintg bier nicht gent trincfen. Benebig,

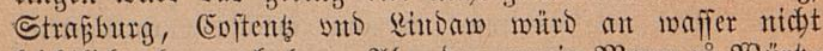

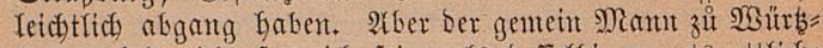
butrg witro nicht fo reid) jein, als Deffelbigen urts ettlidse Dombernt. Den Bayern vno Schmaben witro es mol gefn, wamt fie fein manger an ber notturfft bettent. Die Statt werben lebig pom Tobt jein, jo balo mant fie bat

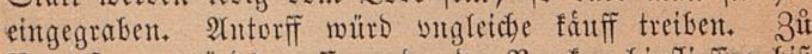

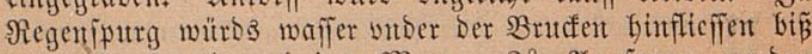
gefn (5onftantinopel ins Meer. Bit 2ugiputrg werbent

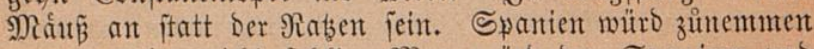
want es jum nidyt fehlt. Man witro Den Spantert vno ben Polen adyt auff bie háno baben múfen. Das Niber= fant würb zuifteigen baben will ea in bas Dberlanto. Die Stalianter werben jebr bie Ejel truten. Die Earacentent werben medytig you ben Mucfen vexiert merben. (5; witrd viel fiid im Meer geben, uno niergent meh fals 
Dan in ber pfanten, vil Sam im Beberlants, vil fant zu

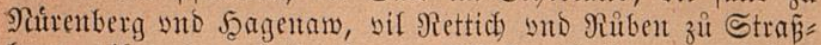

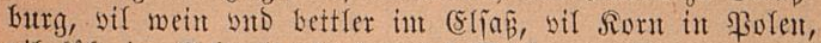
sil fúf im Sdymeitzerlanto, viel Debjen it Bugeren, vil

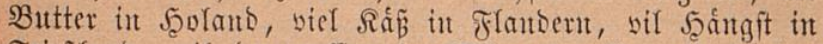

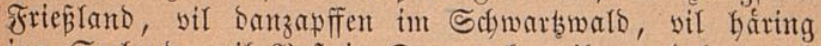
inn Seelants, vil $\Re o \tilde{B}$ in Denmardf, vil mandelent vmb Speir, vil f̧onig in Der Enjel, wil 5opffen in Saxen, viel Specf in Weitphalen, vil Sins im Nobrblingergaw,

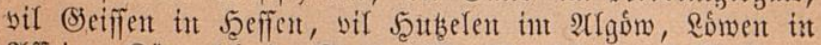
2(ffrica, Rümunich in Malta, Mautbeerbletter zü Meffana.

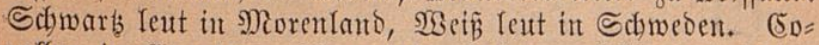
raflen in Egypten, 3immet in 3alon, pfeffer ynto imber in Eaficutb, Sreiffent in Jnbia, Bantbertbier it Partbia, Tigertbier in Sircania, Bertint in \$erjten, Myrrben itt Arabien, Thüc) yon 2̂ntorfi, Siewierbs yon Rifabona, Seiben yout Bentedig, Bardbet yout Błm, Eijenwerdf vont Siüren=

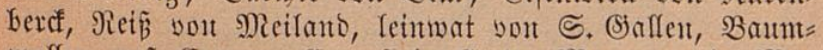

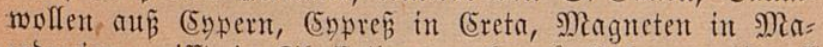
cebonten, giffit in Thefinalien, zucfer zu \$alermo fümpfi in Maflom, feüwr im Sectelberg, Echnee aufi 2tpent= gebingen, Beltluter yom (5humerice, Rantgenwein yon Dant, Gammelfleifo in Maltr, Rüngelein in Spanten,

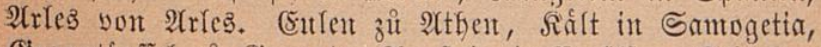

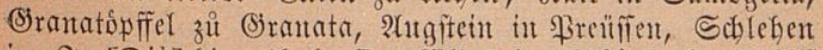

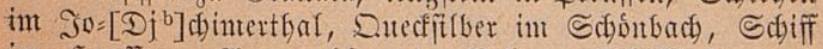

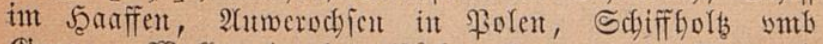
(B)enta, SButlen it ber Bich, Brumten in Burguto

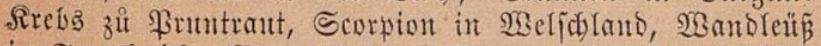
in Frantfreid, Marmelitein zi Berona, Reinfal in Shofte= rich), Sromerd in Eyffland, Edbleiffiteint zit Babentorn, graw Gajen in littbaw, \$̧faffenturent vuno Ejel zü Rom,

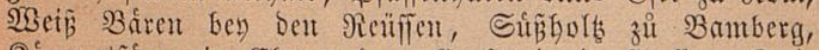

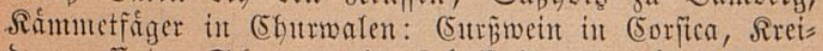

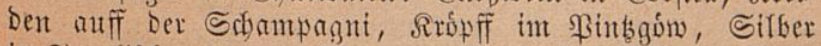
in Tyrofifden bergen, פarm wajier in ben Babern, Salb

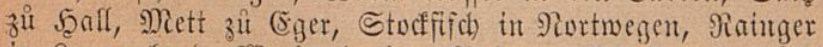

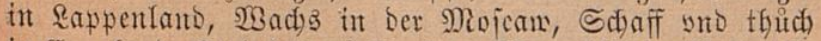
in (Ëtgelanto, Steinjalb zi (Eraffom, פBeyerfiid) in $2 B$ ejterich), 


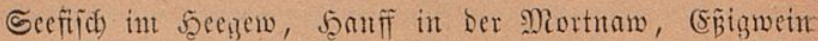
zit Ingelftatt, Eiddelen im Rorbgaw, Danbento perb zü Neaples, Juben zit Jraudfutt, Mutfeltbier in Sarbinien,

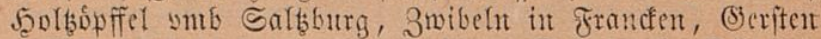
auff Der 2 ab, feiten vmb 5eibelberg, Salmen in Sdyotten vito (B)ind burdi afle Eant.

Rrandfeyten arsuegen ynd ferben.

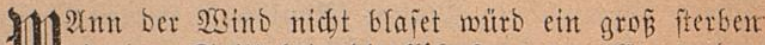
10 in Dem Epittal in bie floh fommen. (Es werden fo vif geiftliche iterben, bas man nientands fïtoen wilto

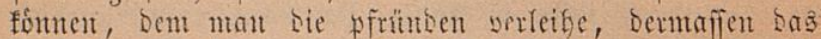
jen vif zwo been, vier nnb mebr befiben werben. Die blinben werben ntidt einten iticfen lefen, bie tauben werbent

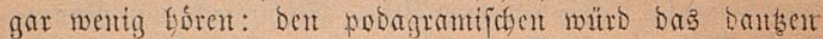
erleiben, bic itummen merben ftillidymeigen wno bie

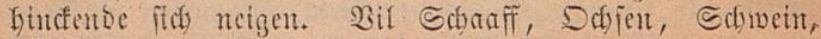

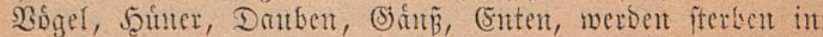
ben futsen, sub wäro ein fold́ iterben nicht nnoer bie

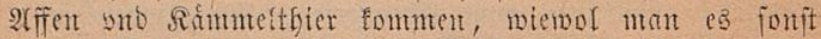
gentaw fitcht, Das auth bie Frofich yund fidnecfent yor grofiem foblecfen nit thieff genig fidser in ber erbent itecfen. Bil wutben werben yntheilbar jein. (sin $2 B u n b a r b$ t ber

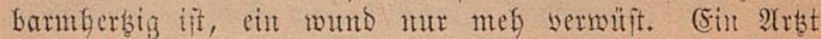
verzagt, ber bie fratcfent viel fragt, fic Doppel ylagt.

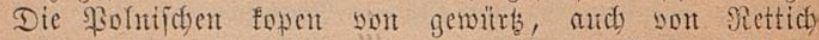
ynto zmibeln bie fürls, werben bent luifit vergifften:

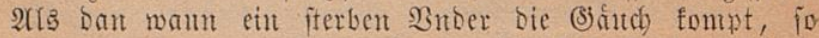

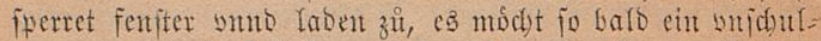

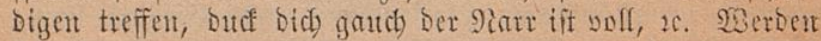
fich bie jungen wie bie alten zü fterben entiebsen. Rein

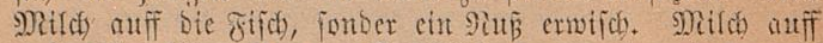
wein ift gifft, 2tber altif SRild Delt weint, bas mag

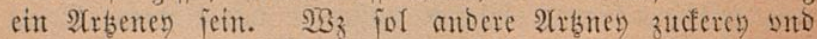

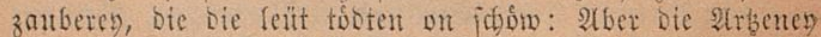

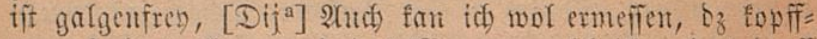
we erforbert efien, vnd das Magenwee idhetifen bas barfi 
untid) fein Theophrajtift weifien. Bno efien nut nit ge=

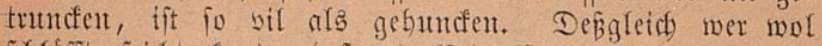

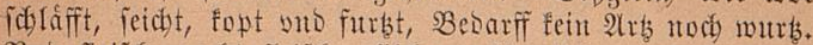

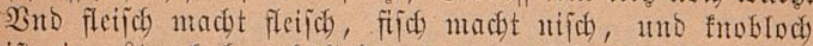
ift ein guter foch, erbalt belt bauten nuch, Den 2 potecfern zit fdmods, who Den Doctorn zit pod). (Ein fappen adst Monat alt, id) fütr ein Reyjers effen balt, wiemol ein Rodberiperger Bautr auth) mit ấ. Die flüt bub Eatarrbi

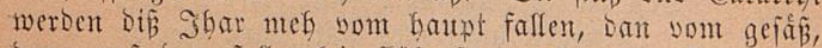

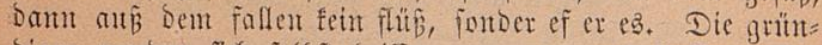
bigen werbent fich jelbit beifien grammen nno freflen. Die blobigfeit oer augent witro Dem gefidyt mee thint. Die pa=

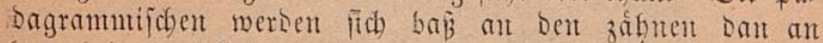
Dent beinen befunden. Die gejunten merben fich beffer gefaben Dan bie francfen. Die Das dntrblantfent triegen, werben offt ben fonigitul Eepithen, buto foll jbit in foldbent auffiratfif erlanbt fein, mant fie fein wife haben, bie finger ober bas bembo zu Erandben, ober, wie ichs non eim lecfer gefehen bab, zieh ben eittent ftumpef aus, vnto

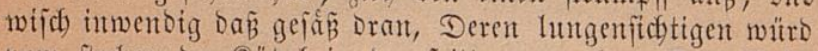
yout ftechen Der Rithel in Der jeitten vergefn. Daß (S)ün

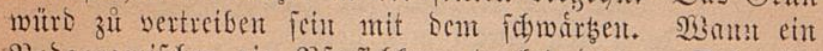
Bobagramificher ein Pferfichern trutcfet Daß en Del gibt, io whirb im gefolffen. Die feltchten getreutuen Siajen

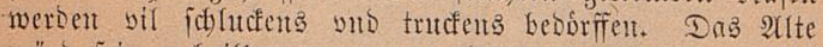
whitri feit vnbeifbar von wegen ber vergangnen jar. (5: whito Das leben foiten benfelbigen Die on fterben vitD als Dan werben fite feit bectelbatb meh beborffen. Surbumb wir minfen vits zålebs alle im fátîlin bebelfifen, Da ber

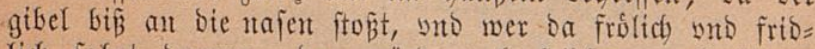
lich fahrt bauton, ber wüto aud fribfam aufferithon. Darauff Dorfift ich ichier iterben, Dá jut aljo jeg), mant es nicht weh thet, ond fein mübst.

Nun bas id) es recht beichlier, baut io bas ento gut ift, fo ift es afles gut, fagt eint mabl

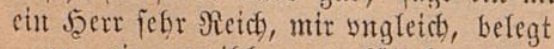

er ein zwildsenen titttel mit borten von gutloenen ftücfent. 
(E3 iteft in Ecclefiajte.

101 (5r alfzeit auff alf winto will jehent, 11. Der wutro nidft fáben ober mábent: Drumb nam ich SBintholo dife mulh, Bnd prodfet bije practicf gie Das bie Stenthlanter vnno fternjountaer Betriegent nidft meh ftatt pund lanber Mit jarich \$rogunoiticationen,

Da fie Den Serten who Rationen restlen vorjagen fünfftig jadben

Bno litgen bas bie bimmel fradsen, WBoflen bie leut mit fternen idsecfent.

Dod) wer wolt glaubent bijen gecfen.

[Dij ${ }^{b}$ ] 2 eil fie vffit in geringen bingent

(Sa) báblich grob zajjamen flingen. Snb jesen offt ein Sibatbenthut, Da wol ein filbghit bejier thit. STa fie in foldhen padjen feblen Wer will fie bann fúr glaubfafft zeglen In ftucfen baran meh gelegen

Sagnt frandfbeyt, frieg fid jollen regen, SWa fie nicht jagen war bem Sauten, TSant or folf pflantien poer Mauren, Wite molten fies ban treffen gleidh

Mit Şern wno jrem fontigreids? Man laß̧t bie Sterntunjt gelten itát Int juer Senteralitet Das ein groj neiguth baut bejuber Die ober Estrper zut ben vntbern, Afber man bandelt viel zì fintwo

In Der particularitet, WBie beut thuำ vnjre Manuiften Mantes junger bes NBiberdifiten, Weldser im jelbjt nidjt fond berfuntent Das ju eitt Berjer nody jolt idsinden. Derbalben bleibt nur uneridbrocfen, WBan fie fobn biffe proctidf proden, 


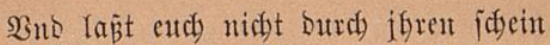
Erletben meber bier nods wein,

(5.3 ift ein Irituolutgium, Butb madyt bie leut mit nidyten frumb. Sonber meî́t fie yon Sott zunt iternen, Das ift, zur ichalen von bem fernen. (S) Iaubt aber bit id) mut vortan

Dem Rechtent gitin erfabruen Manm, Weldser verzudt ijt morben gar Bib it bent Dritten bimmel zwar Der ipridyt, want Sott hie furr vets ift $23 z$ f Das fterten gichóp ni nichts ichaben fant. TSan wir res idhopffers huld mur hat Derbalb auti Das nuan (Sott zit idmod) Sold lopplifh bing nicht galt zu boda,

So bab id bie bie 2 etterbanten Jin ichimpfin ein wentig wollen manen, Das fie jr practicf wno vorjagen Ein wentig Gobeln vno benagen.

Bentagents wol ir metterichmecter Sin fintitern ftern gibts ráje lecfer, Doer wolt jr ben bent juthumb ftácfen So werben ez bie Bantren merten, Snt es nidyt fomten meh yertruffen, Sonber entd) lant int Gintbern gutefen. Da gutf but iterngauth, gucf Du gaud

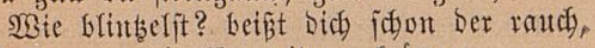
Wholfer mu jr Nemzeitung frimer, Die Brocticf wirb eud angenemer Dan het es Mutnar Nấbod gitelt, Diemeil es entid traigt idsmutsig gelt. Wolfer fautfit jr newzeitung idreyer Sete işt wat numb yom Sternen geber.

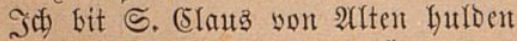
Dab er eud 10000, gutben (Eintonmens jarrlta moll befonerent Dit liegento guter, bie euch nebren. 
Das wer ein bo B́, bas wer git leben, Dieneil wir wart an winichen eben, VQunficht id fo már ben redyten butb, Igetl mir eins wie bas ander mutst, Dod) mant es mar wuro in cim icherts SBie wurb bir Iachen ba Dein berbs, BHo wan es fobut geft binderwerbs

So tît er mur gemulnidut im idberb, SBem nidyt mutro giteren poer berks

(Eey) fro ber bieren ynto bes fterts. Situ bey bem ficwants yno fterts

(Ento fich bie Edants vnt idherb.

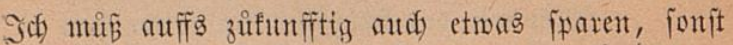
wa id es allez jagt, fo wer es meh bant Das halb: Sono euth bortbin meitter zit= rboten, erwartet bie zufunffit bes Gindentent fotten.

(5. 23. Brocticfprodfer vno Stemtentoder Seinfoldt Seinflit von Rarmutr im Rebelidsiff.

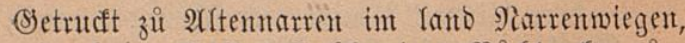
Durd) Den (Eulfus (5od)learicus Büdytrudfer z̊ Narrmeibent.

StUB.Ffm

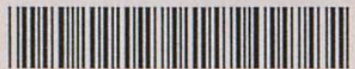

54410891 

